Review

\title{
Optical and Structural Properties of Si Nanocrystals in $\mathrm{SiO}_{2}$ Films
}

\author{
Timur Nikitin and Leonid Khriachtchev * \\ Department of Chemistry, University of Helsinki, P.O. Box 55, FI-00014 Helsinki, Finland; \\ E-Mail: timnik@eumx.net
}

* Author to whom correspondence should be addressed; E-Mail: leonid.khriachtchev@helsinki.fi; Tel.: +358294150310.

Academic Editor: Lorenzo Rosa

Received: 4 March 2015 / Accepted: 10 April 2015 / Published: 22 April 2015

\begin{abstract}
Optical and structural properties of Si nanocrystals (Si-nc) in silica films are described. For the $\mathrm{SiO}_{x}(x<2)$ films annealed above $1000^{\circ} \mathrm{C}$, the Raman signal of Si-nc and the absorption coefficient are proportional to the amount of elemental Si detected by X-ray photoelectron spectroscopy. A good agreement is found between the measured refractive index and the value estimated by using the effective-medium approximation. The extinction coefficient of elemental $\mathrm{Si}$ is found to be between the values of crystalline and amorphous Si. Thermal annealing increases the degree of Si crystallization; however, the crystallization and the $\mathrm{Si}-\mathrm{SiO}_{2}$ phase separation are not complete after annealing at $1200{ }^{\circ} \mathrm{C}$. The 1.5-eV PL quantum yield increases as the amount of elemental Si decreases; thus, this PL is probably not directly from Si-nc responsible for absorption and detected by Raman spectroscopy. Continuous-wave laser light can produce very high temperatures in the free-standing films, which changes their structural and optical properties. For relatively large laser spots, the center of the laser-annealed area is very transparent and consists of amorphous $\mathrm{SiO}_{2}$. Large $\mathrm{Si}-\mathrm{nc}$ (up to $\sim 300 \mathrm{~nm}$ in diameter) are observed in the ring around the central region. These Si-nc lead to high absorption and they are typically under compressive stress, which is connected with their formation from the liquid phase. By using strongly focused laser beams, the structural changes in the free-standing films can be made in submicron areas.
\end{abstract}

Keywords: Si nanocrystal ( $\mathrm{Si}-\mathrm{nc}) ; \mathrm{SiO}_{2}$ film; Raman spectroscopy; photoluminescence; laser annealing 


\section{Introduction}

Many limitations of modern electronic devices may be overcome by implementing photonics into electronics [1-4]. Integration of Si-based photonics with CMOS technology is a promising approach because it gives the possibility to merge electronics and photonics in the same chip [5-9]. Many optical functions such as, for example, light sources, amplifiers, waveguides, modulators, memory, and detectors should be achieved in order to fulfill this integration. Realization of a true monolithically fabricated injection Si laser with a small size is a particularly challenging task. The fundamental problem is the low light-emitting efficiency of bulk silicon due to its indirect band gap $(\sim 1.1 \mathrm{eV})$, which leads to long radiative times $(\sim \mathrm{ms})$ and therefore, mostly non-radiative recombination of the excited carriers. The efficiency of the light emission can be enhanced by increasing the overlap of the wave functions of the electron and hole via, for example, spatial confinement [10-13]. The unusual properties of Si structures are observed when the size is less than the free exciton Bohr radius of $4.3 \mathrm{~nm}$ in bulk Si. This quantum confinement (QC) effect leads to the following main changes in the material properties: (i) an increase of the radiative probability due to delocalization of the wave functions in the momentum space, which increases the electron-hole wave function overlap and (ii) a shift in the emission wavelength to the visible region due to an increase of the band gap, which is controlled by the Si nanostructure size. Moreover, the number of sites within the volume of $\mathrm{Si}$ nanostructures, where non-radiative recombination can occur, decreases considerably. It should be mentioned that understanding electrical transport mechanisms in the systems considered below is very important for many applications [14]; however, this topic is outside the scope of the present review.

Porous $\mathrm{Si}$ is the first example of a Si-based material with enhanced luminescence efficiency. Strong room-temperature photoluminescence (PL) in visible spectral region from this material was discovered by Leigh Canham in 1990 [15]. Visible luminescence ranging from green to red in color was soon reported by Canham et al. [16] for other porous-Si samples and by other researchers [17,18] and ascribed to the quantum effects in Si structures with a size of $\sim 3 \mathrm{~nm}$. The blue shift of the PL and optical absorption with the increasing porosity (decreasing Si nanocluster sizes) provided the first important evidence that the QC plays an important role in light emission from porous Si. This blue shift is a result of the band gap expansion controlled by the size of Si nanostructures $[13,19]$.

It was soon understood that oxidation of small Si crystallites has a great influence on the light emission [20,21]. The study by Wolkin et al. of oxidized porous Si has shown that the light-emitting centers can involve the $\mathrm{Si}=\mathrm{O}$ covalent bonds located on the crystallite surface [22]. For oxygen-passivated $\mathrm{Si}$ crystallites, a stabilized electronic surface state is formed on a $\mathrm{Si}=\mathrm{O}$ covalent bond and various recombination mechanisms can operate depending on the crystallite size. For larger sizes $(>3 \mathrm{~nm})$, recombination occurs via free excitons since the band gap is not wide enough to stabilize the $\mathrm{Si}=\mathrm{O}$ surface state. For intermediate sizes $(\sim 2.5 \mathrm{~nm})$, recombination involves a trapped electron localized on the $\mathrm{Si}$ atom of the $\mathrm{Si}=\mathrm{O}$ bond and a free hole. For smaller sizes $(<2 \mathrm{~nm})$, recombination occurs via trapped excitons.

However, QC has been demonstrated for a number of Si nanomaterials. In contrast with oxygen passivation, for hydrogen-passivated porous $\mathrm{Si}$, recombination occurs via free exciton states for all crystallite sizes and follows the expected QC behavior [22]. Ledoux et al. studied Si nanocrystals (Si-nc) prepared by pulsed $\mathrm{CO}_{2}$ laser pyrolysis of silane in a gas flow reactor and deposited on a 
substrate [23]. It was observed that the PL band was blue-shifted as the size of Si-nc decreased from 8 to $2.5 \mathrm{~nm}$. This dependence clearly follows the QC model. The QC mechanism operates also for alkane-terminated Si-nc. Hannah et al. [24] convincingly demonstrated using pressure-dependent PL studies that the PL arises from the core-states of Si-nc with indirect-gap transitions. The same PL mechanism has also been reported to operate for Si-nc in silicon-nitride films $[25,26]$.

Another attractive Si-based material, which is the focus of the present article, is composed of Si-nc embedded in a $\mathrm{SiO}_{2}$ matrix. This material is chemically and mechanically more stable compared to porous $\mathrm{Si}$ and also emits light in the visible region. Si-nc in silica films can be prepared by various methods such as, for example, molecular beam deposition (MBD) [27,28], Si-ion implantation [29-32], sputtering [33-39], plasma enhanced chemical vapor deposition (PECVD) [33,34,40-42], low pressure chemical vapor deposition with subsequent thermal oxidation [43-45], reactive evaporation of $\mathrm{SiO}$ powder in oxygen atmosphere [46], and electron beam deposition [47]. Two types of architectures are most commonly prepared: (i) Si-rich silicon oxide $\mathrm{SiO}_{x}(x<2)$ films [31,33,40,48,49] and (ii) $\mathrm{SiO}_{x} / \mathrm{SiO}_{2}$ [37,46,47,50-53] and $\mathrm{Si} / \mathrm{SiO}_{2}$ [27,28,35,54-57] superlattices (SLs). Si-nc are formed in these materials by furnace annealing above $1000{ }^{\circ} \mathrm{C}$. Studies of $\mathrm{Si}-\mathrm{nc}$ in $\mathrm{SiO}_{2}$ have been particularly stimulated by the observation of optical gain in this material [58,59]. In addition to PL, electroluminescence in visible region from porous $\mathrm{Si}$ and from $\mathrm{Si}-\mathrm{nc}$ in $\mathrm{SiO}_{2}$ has been reported [60,61].

The origin of light emission from $\mathrm{Si}-\mathrm{nc}$ in a $\mathrm{SiO}_{2}$ matrix is still controversial. It seems that the conclusions on the mechanism of the light emission from oxidized porous Si [20-22,62] are relevant to Si-nc in $\mathrm{SiO}_{2}$. Similarly, the PL of Si-nc (sizes $>3 \mathrm{~nm}$ ) in $\mathrm{SiO}_{2}$ can be ascribed to the QC effect [63-66]. On the other hand, the importance of the $\mathrm{Si}-\mathrm{nc} / \mathrm{SiO}_{2}$ interface in the light-emitting properties has been stressed [31,35,67,68] and, in particular, the defect origin of the PL has been discussed [27,29,31,69-71]. Godefroo et al. have convincingly demonstrated that defects are the dominant source of the 1.5-eV PL [52]. In their experiment, an amorphous $\mathrm{SiO} / \mathrm{SiO}_{2} \mathrm{SL}$ grown by reactive evaporation of $\mathrm{SiO}$ powders in an oxygen atmosphere was thermally annealed at $1100{ }^{\circ} \mathrm{C}$ for $1 \mathrm{~h}$ under $\mathrm{N}_{2}$ atmosphere to produce Si-nc. The authors could switch the PL mechanism between the QC and defect mechanisms by passivation with hydrogen and ultraviolet illumination, respectively.

Optoelectronic applications of Si-based materials require knowledge of their optical and structural properties. For $\mathrm{SiO}_{x}(x<2)$ films prepared by PECVD, the energy-filtered transmission electron microscopy (EFTEM) show that the $\mathrm{Si}-\mathrm{SiO}_{2}$ phase separation starts after annealing at $900{ }^{\circ} \mathrm{C}$ for $1 \mathrm{~h}$ under $\mathrm{N}_{2}$ atmosphere when Si clusters become visible in an oxide matrix [72]. The dark-field (DF) transmission electron microscopy (TEM) technique reliably shows that the Si clusters are amorphous for annealing temperatures of $900-1000{ }^{\circ} \mathrm{C}$, and their crystallization begins at $1100{ }^{\circ} \mathrm{C}$. Higher annealing temperatures promote further crystallization of the Si clusters. The Si-nc size as a function of the annealing temperature and $\mathrm{Si}$ content has been studied for samples prepared by different deposition methods. For samples prepared by PECVD, the TEM results indicate that the Si-nc sizes increase with the increasing annealing temperature for a given Si content and with the Si content for a given annealing temperature [40]. The increase of the Si-nc sizes with the annealing temperature is also observed for $\mathrm{SiO}_{x}$ films grown by magnetron sputtering [37]. For $\mathrm{SiO}_{x}$ films prepared by ion implantation, the nucleation and pure growth stages of the nanocrystal population are almost over after 1 min of annealing at $1100^{\circ} \mathrm{C}$ in $\mathrm{N}_{2}$ [31]. For longer annealing periods, the sizes of Si-nc increase due to coalescence or Ostwald ripening process $[31,73]$. 
Several methods have been employed to study optical constants of different materials containing Si-nc. These methods include, for example, optical ellipsometry [74-76] and $m$-line measurements [77-79]. The analysis of the optical properties often uses the Bruggeman effective-medium approximation [80-82]. The Tauc-Lorentz model provides optical constants of Si-nc deposited on a substrate [82]. The results show that the optical properties of Si-nc are quite different from those of amorphous and crystalline $\mathrm{Si}$. It has been found that at a given wavelength, the refractive index of $\mathrm{SiO}_{x}$ films increases for larger Si-nc for Si-ion implanted samples [74] and for larger Si content for PCVD samples [75]. Chen et al. have developed a quantitative approach of obtaining depth profiles of the optical constants [81]. Moreno et al. report that the refractive index of Si-nc is lower than that of amorphous and crystalline bulk Si [80]. They also conclude that the refractive index is rather independent of the Si clusters sizes $(3.6-4.6 \mathrm{~nm})$ and is mostly affected by the degree of crystallinity. For $\mathrm{SiO}_{x} / \mathrm{SiO}_{2}$ SLs prepared by magnetron sputtering, negative optical birefringence ( 1\%) was observed, originating from the periodical set of parallel planes of two different materials constituting the SL [77,78]. At a given wavelength, the absorption coefficient of annealed $\mathrm{SiO}_{x}$ films prepared by magnetron sputtering and PECVD increases with the Si content $[33,76,83]$. The increase of the Si-nc mean diameter leads to an increase of absorption in porous $\mathrm{Si}$ [84] and in $\mathrm{SiO}_{x}$ films prepared by ion implantation [80]. For a fixed Si content, the absorption coefficient of films prepared by magnetron sputtering and PECVD decreases with the increasing annealing temperature, which is due the amorphous-to-crystalline transition of Si in the samples [33].

Another method of measuring the optical properties of $\mathrm{SiO}_{x}$ films is based on the PL-filtering effect. A silicon oxide layer containing Si-nc on a silica substrate forms a planar waveguide. Spectral filtering of the PL occurs when the PL is detected from the waveguide edge along the film surface. This effect has been found for $\mathrm{Si} / \mathrm{SiO}_{2} \mathrm{SLs}$ [57] and for $\mathrm{SiO}_{x}$ films $(x<2)$ on silica substrate [79,85-87]. The detailed description of this effect is presented later.

The Tauc relation [88] allows one to estimate the band gap of $\mathrm{SiO}_{x}$ films containing Si-nc $[31,33,36,44,74,89,90]$. A study of Si-nc deposited on a silica substrate shows that the electronic band structure of Si-nc is quite different from that of bulk silicon and that the band gap increases as the Si-nc size decreases [82]. The latter observation is explained by the QC effect. The increase of the band gap for smaller $\mathrm{Si}-\mathrm{nc}$ is also observed for implanted $\mathrm{SiO}_{x}$ samples [31,74]. The absorption threshold is blue-shifted for smaller Si-nc, which indicates the QC effect on the Si-nc band gap $[33,36,84,90,91]$.

Laser annealing is an interesting approach to change the structural and optical properties of Si-based materials. The heating effect of pulsed radiation is especially strong [92]. However, it can also be substantial for continuous-wave (CW) irradiation of free-standing porous Si films [93]. A similar heating effect of $\mathrm{CW}$ laser radiation is observed in free-standing $\mathrm{SiO}_{x}$ films leading to a strong increase of the Raman signal of Si-nc [56,94-96]. Si-nc prepared by laser annealing show high compressive stress ( $\sim 3 \mathrm{GPa}$ ), which is evidenced by an up-shift of the Raman band $[56,96]$. The compressive stress is formed in a solid $\mathrm{SiO}_{2}$ matrix when the volume of $\mathrm{Si}-\mathrm{nc}$ suddenly increases after its crystallization from the liquid phase. The high stress can be relaxed by irradiating the stressed Si-nc with lower laser power [56]. Free-standing films are used to obtain the above described laser-induced effects because for films on substrates the laser-induced heat is reduced due to thermal flux to substrate [97]; however, it is still observable [98]. 
In agreement with the experimental observations, the theoretical simulations of isolated hydrogen-passivated Si nanostructures show that the band gap and the PL peak position change according to QC [13,19]. The behavior of these parameters is different for oxidized Si-nc [99]. Theoretical studies highlight the importance of the interface region between Si-nc and a $\mathrm{SiO}_{2}$ matrix for the light-emitting properties [100,101]. The absorption edge tends to be red-shifted due to the formation of the $\mathrm{Si}=\mathrm{O}$ or $\mathrm{Si}-\mathrm{O}-\mathrm{Si}$ bonds at the $\mathrm{Si} / \mathrm{SiO}_{2}$ interface [102-104]. The presence of oxygen atoms bonded to the surface of Si-nc as well as deformation of Si-nc are found to influence the optical band gap [105]. Luppi et al. have considered small Si-nc (up to $1 \mathrm{~nm}$ in diameter) by applying $a b$ initio calculations [106]. In the case of the $\mathrm{Si}-\mathrm{O}-\mathrm{Si}$ bridge bond at the cluster surface, an emission peak at about $1.5 \mathrm{eV}$ is obtained. In accord, the PL in this region is often observed experimentally from silica films containing Si-nc. The calculated emission peak is red-shifted with respect to the absorption, which is also in agreement with the experimentally observed Stokes shifts between the absorption and PL spectra.

Theoretical calculations show that the surrounding matrix can produce some strain on Si-nc [107-109], and this strain depends on the oxidation degree [110,111]. The magnitude of strain affects the band gap, which shows an oscillating behavior with the Si-nc size, not strictly following the QC rule [110]. Moreover, the disorder of nanoclusters has a large effect on their optoelectronic properties [112,113]. In particular, amorphization reduces the optical band gap and increases the absorption strength in the visible range.

Guerra and Ossicini have calculated the recombination rates for different classes of Si-nc in the diameter range of $0.2-1.5 \mathrm{~nm}$ [114]. The authors also consider different conditions of passivation, strain, and symmetry in order to find the best conditions of radiative emission. As a result, they have found that the smallest, highly oxidized, crystalline clusters are the most optically active $\mathrm{Si} / \mathrm{SiO}_{2}$ structures.

\section{Results and Discussion}

\subsection{Furnace-Annealed Films}

In this section, we describe the properties of $\mathrm{SiO}_{x}(x<2)$ films on silica substrates for different annealing temperatures $\left(400-1200{ }^{\circ} \mathrm{C}\right.$ ) and $\mathrm{Si}$ contents $\left(x\right.$ from $\sim 1.3$ to $\sim 1.98$ ) [115-118]. The $\mathrm{SiO}_{x}$ films (thickness from $\sim 1.5$ to $\sim 2.5 \mu \mathrm{m}$ ) are deposited on silica substrates by MBD. The MBD samples are compared with samples prepared by ion implantation [118].

\subsubsection{Correlation between Optical and Structural Properties}

The as-prepared MBD films (annealed at $400{ }^{\circ} \mathrm{C}$ for better mechanical stability) with substantial Si content show a broad Raman band at $\sim 470 \mathrm{~cm}^{-1}$, which is characteristic of amorphous $\mathrm{Si}$ (Figure 1) $[27,48,55,119,120]$. After annealing above $1000{ }^{\circ} \mathrm{C}$, crystallization of the amorphous Si inclusions occurs as evidenced by the narrowing and upshift of the Raman band to $\sim 518 \mathrm{~cm}^{-1}$ (Figure 1). When measured with low laser intensity, the Raman band position $\left(518-519 \mathrm{~cm}^{-1}\right)$ is rather independent of the Si content and of the annealing temperature in the $1100-1200{ }^{\circ} \mathrm{C}$ range. According to the phonon confinement model [121,122], this result suggests that the Si-nc sizes evidenced by 
Raman spectroscopy do not change much under these conditions. However, growth of Si-nc with increasing annealing temperature was observed for $\mathrm{SiO}_{x}$ films prepared by other deposition methods $[37,40]$.

For $\mathrm{MBD} \mathrm{SiO}_{x}$ films with very small excess of $\mathrm{Si}(x>1.9)$, the Raman bands of amorphous and crystalline Si are nearly invisible [116], in qualitative agreement with the earlier results [27]. The reason for this observation is the small amount of properly coordinated $\mathrm{Si}$ atoms ( $\mathrm{Si}$ atoms bonded to four "bulk" Si atoms) for samples with low Si contents. A similar explanation was applied in a study of amorphous $\mathrm{Si} / \mathrm{SiO}_{2} \mathrm{SLs}$, where the Raman bands of amorphous Si were observed for thicker Si layers ( $\geq 2 \mathrm{~nm}$ ) and the Raman-scattering cross section decreased for thinner Si layers [55,123].

As the annealing temperature increases, the low-frequency shoulder in the Raman spectra responsible for disordered Si and/or small Si clusters gradually decreases (Figure 1). However, even after annealing at $1200{ }^{\circ} \mathrm{C}$, the low-frequency component of the Raman band does not disappear and its area is comparable to that of the high-frequency component. As a possibility, small Si grains in a $\mathrm{SiO}_{2}$ matrix may be disordered even after annealing at this temperature whereas larger Si grains are crystallized.

X-ray photoelectron spectroscopy (XPS) confirms that the structural reorganization is not complete after furnace annealing (Figure 2). The $\mathrm{Si}-\mathrm{SiO}_{2}$ phase separation increases with the annealing temperature, which is evidenced by the increasing amount of elemental $\mathrm{Si}$ and $\mathrm{SiO}_{2}$ and the decreasing amount of SiO. However, a large proportion of suboxides $(10-15$ at. $\%$ for $x=1.7)$ is still detected after annealing at $1200{ }^{\circ} \mathrm{C}$ [116]. This result indicates incomplete $\mathrm{Si}-\mathrm{SiO}_{2}$ phase separation in these materials.

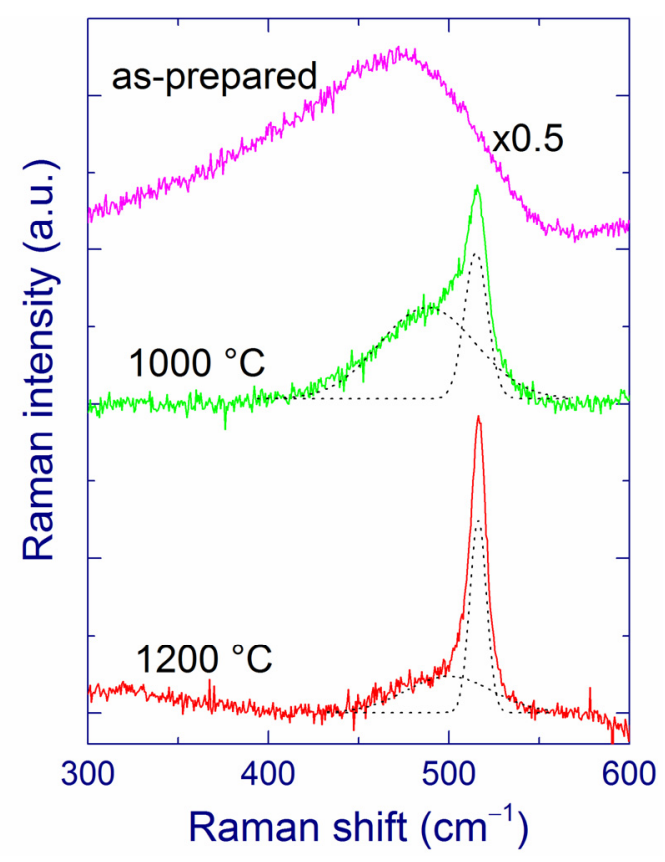

Figure 1. Raman spectra of a $\mathrm{SiO}_{1.75}$ film as-prepared and annealed at 1000 and $1200{ }^{\circ} \mathrm{C}$ (fitted by two Gaussians). The spectra are vertically shifted for better presentation. The

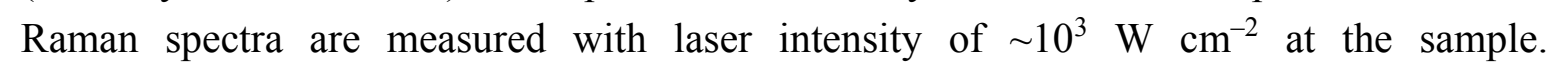
Reproduced with permission from [116]. Copyright 2012, AIP Publishing LLC. 


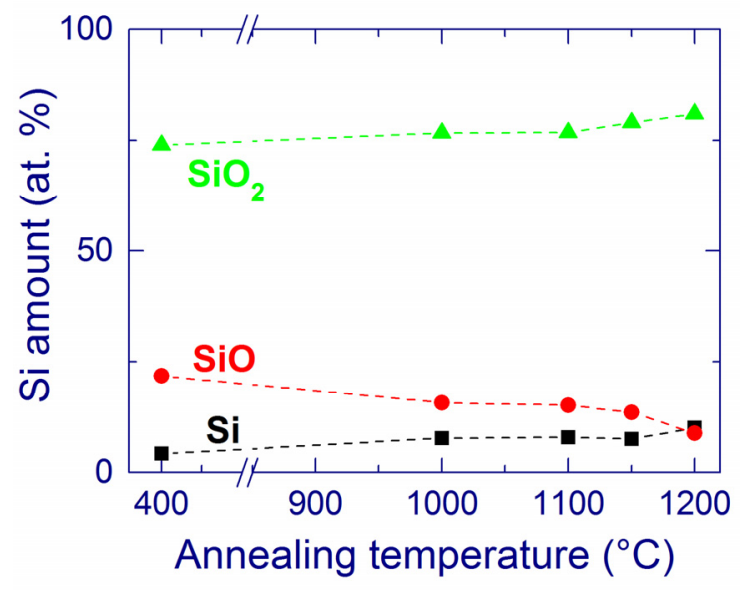

Figure 2. Composition of a $\mathrm{SiO}_{1.7}$ film provided by X-ray photoelectron spectroscopy (XPS) as a function of the annealing temperature. Reproduced with permission from [116]. Copyright 2012, AIP Publishing LLC.

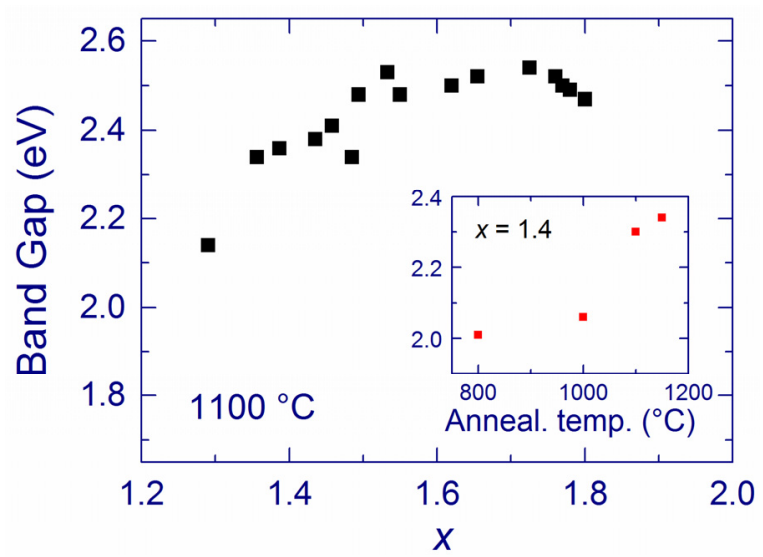

Figure 3. Band gap of $\mathrm{SiO}_{x}$ films annealed at $1100{ }^{\circ} \mathrm{C}$ as a function of $x$. The inset shows the band gap of a $\mathrm{SiO}_{1.4}$ film as a function of the annealing temperature. Reproduced with permission from [116]. Copyright 2012, AIP Publishing LLC.

Additional information about the properties of $\mathrm{SiO}_{x}$ films can be obtained from the band gap. For a given annealing temperature (from 900 to $1200{ }^{\circ} \mathrm{C}$ ), the band gap of $\mathrm{SiO}_{x}$ films increases as the $\mathrm{Si}$ content decreases [116]. For annealed at $1100{ }^{\circ} \mathrm{C}$, the band gap increases from 2.2 to $2.6 \mathrm{eV}$ as $x$ increases from 1.3 to 1.8 (Figure 3), meaning that the Si-nc sizes somewhat decrease. According to the ab initio calculations for Si-nc with oxygen bonds at the $\mathrm{Si} / \mathrm{SiO}_{2}$ interface, the obtained band gap change (2.2-2.6 eV) corresponds only to a marginal change of the Si-nc sizes, the average size being $\sim 1 \mathrm{~nm}$ [124]. On the other hand, the position of the maximum of the Raman bands at $518-519 \mathrm{~cm}^{-1}$ corresponds to larger sizes (3-4 nm) [121,122], and this value is supported by TEM measurements of similar materials [125]. These different estimates can be connected with low sensitivity of the Raman and TEM methods to very small Si grains. The low-frequency Raman scattering at $490-500 \mathrm{~cm}^{-1}$ can be contributed by smaller Si-nc (1-2 $\mathrm{nm}$ ) that may be partially disordered, which would agree with the Si-nc sizes derived from the band gaps. It should also be mentioned that the band gap of Si clusters is a complex function of a number of parameters in addition to the size, such as the crystallinity, strain due 
to the $\mathrm{SiO}_{2}$ matrix, and degree of oxidation [110,112]. Moreover, the Tauc law is generally applicable for amorphous semiconductors with absorption coefficients $>10^{4} \mathrm{~cm}^{-1}$ [88]. Thus, the band gaps obtained by applying the Tauc law to crystalline Si grains with lower absorption may be somewhat inaccurate.

The band gap of $\mathrm{SiO}_{x}(x \sim 1.4$ [116] and $x \sim 1.8$ [118]) films increases with the annealing temperature (see the inset in Figure 3 ). This increase can be explained by amorphous-to-crystalline transitions of Si clusters rather than by a decrease of their sizes, following the discussions by Mirabella et al. [33]. Indeed, the Si-nc sizes should rather increase with the annealing temperature $[40,42]$ even though this increase for the MBD samples seems to be minor as suggested by the Raman spectra.

By varying the Si content and annealing temperature, one can control the refractive index and the absorption coefficient of $\mathrm{SiO}_{x}$ films. For a given annealing temperature, the refractive index increases with the Si content (Figure 4a) [118] as also reported previously [49,79]. Extrapolation of the data to $x=2$ yields the refractive index of silica (1.456), which is a good verification of our results. The obtained values are also in agreement with other experimental results for annealing at $1100{ }^{\circ} \mathrm{C}$, $\lambda=632 \mathrm{~nm}$, and $x \sim 1.38-1.63$ [80]. For a given $x$, the refractive index typically decreases upon annealing at temperatures from 1000 to $1100{ }^{\circ} \mathrm{C}$ but increases in the $1100-1200{ }^{\circ} \mathrm{C}$ annealing temperature range [116].

The measured refractive index is interesting to compare with the values derived from the effective medium approximation and the chemical compositions measured by XPS (Figure $4 \mathrm{a}$ for $1100{ }^{\circ} \mathrm{C}$ and in Table 1 for $x \sim 1.75$ ). The agreement between the theory and the experiment is good. The observed small discrepancy can be due to the limitations of the used model (see Section 3.3). It is also possible that the refractive index of nanostructures is different from that of the bulk materials. Finally, in the XPS analysis, the mixture of different suboxides constitutes the SiO component, and this can also contribute to the error.
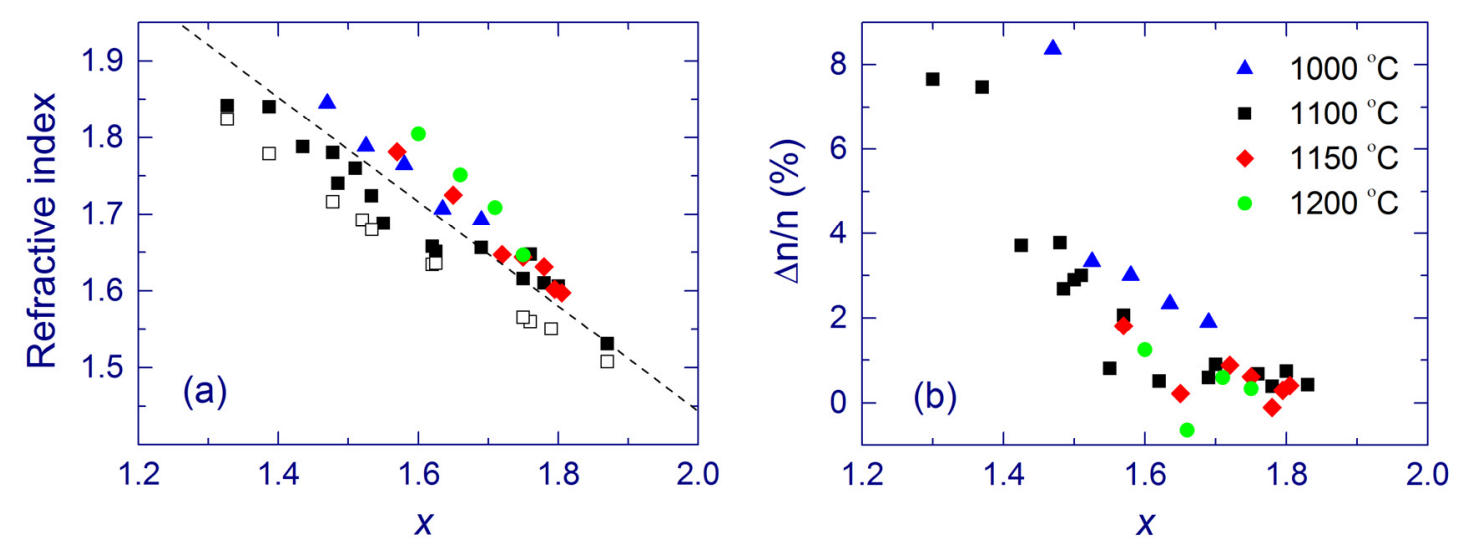

Figure 4. (a) $\mathrm{TE}$ refractive index and (b) birefringence of $\mathrm{SiO}_{x}$ films annealed at $1000{ }^{\circ} \mathrm{C}$ (triangles), $1100{ }^{\circ} \mathrm{C}$ (squares), $1150{ }^{\circ} \mathrm{C}$ (diamonds), and $1200{ }^{\circ} \mathrm{C}$ (circles) as a function of $x$. The open squares show the refractive index obtained using the effective medium approximation and the XPS results for annealing at $1100{ }^{\circ} \mathrm{C}$. The dashed line is a guide for the eye. (a) is reproduced with permission from [116]. Copyright 2012, AIP Publishing LLC. 
Table 1. Experimental refractive index $n_{\exp }$ and absorption coefficient $\alpha_{\exp }$ of a $\mathrm{SiO}_{1.75}$ film for different annealing temperatures $T_{\text {ann }}$ and the estimates for the refractive index $n_{\text {est }}$ and extinction coefficient $k_{\text {est }}$ from the effective medium approximation. Reproduced with permission from [116]. Copyright 2012, AIP Publishing LLC.

\begin{tabular}{cccccc}
\hline $\boldsymbol{T}_{\text {ann }}\left({ }^{\circ} \mathbf{C}\right)$ & $\boldsymbol{n}_{\exp }$ & $\boldsymbol{\alpha}_{\exp }\left(\mathbf{1 0}^{\mathbf{4}} \mathbf{c m}^{-\mathbf{1}}\right)$ & $\boldsymbol{n}_{\text {est }}$ & $\boldsymbol{k}_{\text {est }}(\mathbf{S i O})$ & $\boldsymbol{k}_{\text {est }}(\mathbf{S i})$ \\
\hline 400 & - & 1.57 & 1.575 & 0.45 & 1.25 \\
900 & - & 0.56 & 1.57 & 0.07 & 1.25 \\
1000 & $1.64^{*}$ & $0.134^{*}$ & $1.56^{*}$ & 0 & 0.77 \\
1100 & 1.61 & 0.126 & 1.56 & 0 & 0.53 \\
1150 & 1.64 & 0.120 & 1.565 & 0 & 0.535 \\
1200 & 1.67 & 0.128 & 1.57 & 0 & 0.48 \\
\hline
\end{tabular}

* Obtained by extrapolation of the data to $x=1.75$.

The $\mathrm{SiO}_{x}$ film material prepared by MBD is found to be birefringent [79]. The classical theory of an isotropic asymmetrical planar waveguide predicts that the cut-off positions for the TE modes (with polarization parallel to the film) are at longer wavelengths than the TM cut-off positions (with polarization perpendicular to the film) $[126,127]$. However, the opposite order between the TE and TM peaks is found for some samples (see Figure 20a in Section 3.3). This reversed order of the TE and TM peaks is explained assuming positive optical birefringence, which is connected with the non-spherical shape of Si-nc [79]. Non spherical Si-nc have been observed in similar materials using the combination of electron tomography with plasmon-filtered microscopy [128]. It appears that that the optical birefringence is higher for $\mathrm{SiO}_{x}$ films with the higher $\mathrm{Si}$ content and for lower annealing temperature (Figure 4b) [79,116]. Similar values of birefringence are obtained by $m$-line measurements [115].

The absorption coefficient of $\mathrm{SiO}_{x}$ films can be analyzed using the effective medium approximation and the XPS data (Table 1). For an as-prepared $\mathrm{SiO}_{1.75}$ film, the extracted extinction coefficient of the $\mathrm{SiO}$ phase (0.45) is much larger than the value known for bulk $\mathrm{SiO}(0.055)$ if the extinction coefficient of amorphous Si $(k=1.25)$ is used for elemental Si. This mismatch would be smaller if the amount of elemental Si was underestimated by XPS and/or the extinction coefficient of nanoscale amorphous Si was larger than that of bulk amorphous Si. For annealing at $900{ }^{\circ} \mathrm{C}$, our estimates agree with the extinction coefficients of bulk $\mathrm{SiO}$ and amorphous $\mathrm{Si}$. For films annealed above $1000{ }^{\circ} \mathrm{C}$, the $\mathrm{SiO}$ component is found to be very transparent, in contrast to bulk $\mathrm{SiO}$, and this difference is possibly connected with ultra-small sizes of the suboxide regions.

As the annealing temperature increases, the estimated extinction coefficient of elemental $\mathrm{Si}$ decreases (Table 1), which is interpreted in terms of gradual crystallization of Si [115,116]. However, this process in not complete even after annealing at $1200{ }^{\circ} \mathrm{C}$, because the calculated extinction coefficient ( 0.48 for $x=1.75)$ is much larger than that of crystalline $\mathrm{Si}(0.079)$; thus, Si is partially disordered, in agreement with the earlier results [42]. This "disordered" Si should differ from "ordinary" amorphous Si because no Raman band at $470 \mathrm{~cm}^{-1}$ is present for the films annealed above $1000{ }^{\circ} \mathrm{C}$. Instead, the disordered Si grains may contribute to the Raman scattering at $490-500 \mathrm{~cm}^{-1}$, which is observed in Raman spectra of $\mathrm{SiO}_{x}$ films annealed up to $1200{ }^{\circ} \mathrm{C}$ (Figure 1). As a possibility, ultra-small $\mathrm{Si}$ grains in a $\mathrm{SiO}_{2}$ matrix may be disordered even after the annealing whereas larger $\mathrm{Si}$ grains are crystallized. The presence of this disordered Si may explain the surprisingly large absorption 
of the annealed $\mathrm{SiO}_{x}$ films. Moreover, the optical properties of bulk and nanoscale Si may differ, and the absorption by $\mathrm{SiO}$ inclusions cannot be completely excluded. This explanation of the relatively large absorption coefficient of annealed $\mathrm{SiO}_{x}$ films is quite speculative, and additional experimental data are required to draw more accurate conclusions.

The absorption coefficient and the integrated Raman intensity of Si-nc are nearly proportional to the amount of elemental Si obtained by XPS (see Figure 5 for absorption) [115,116], in agreement with the earlier results [48]. It follows that elemental Si determines the absorption coefficient and the Si-nc Raman signal. A similar conclusion is made for $\mathrm{SiO}_{x}$ films deposited by magnetron sputtering [129]. On the other hand, we have obtained that the Raman scattering cross-section of elemental $\mathrm{Si}$ in $\mathrm{SiO}_{x}$ films prepared by MBD is about three times smaller compared to that of crystalline Si [116]. This difference can be explained by several factors. Ultra-small Si-nc (diameters $<2 \mathrm{~nm}$ ) may be invisible by Raman spectroscopy due to the low proportion of "properly" coordinated Si atoms. The interaction with a $\mathrm{SiO}_{2}$ matrix can change the coordination of $\mathrm{Si}-\mathrm{nc}$, and this effect is stronger for smaller $\mathrm{Si}$ particles. In principle, the broad band gap of Si-nc may affect the Raman cross-section due to the resonance effect. Finally, the complex character of interaction of light with $\mathrm{Si}-\mathrm{nc}$ embedded in $\mathrm{SiO}_{2}$ is not well understood. The present results are not enough to give a more reliable interpretation of these observations.



Figure 5. Absorption coefficient at $488 \mathrm{~nm}$ for annealing at $1000{ }^{\circ} \mathrm{C}$ (triangles), $1100{ }^{\circ} \mathrm{C}$ (squares), $1150{ }^{\circ} \mathrm{C}$ (diamonds), and $1200{ }^{\circ} \mathrm{C}$ (circles) as a function of the amount of elemental $\mathrm{Si}$. The dashed line is a guide for the eye. Reproduced with permission from [116]. Copyright 2012, AIP Publishing LLC.

\subsubsection{Photoluminescence of the MBD Samples}

For $\mathrm{SiO}_{x}$ films annealed up to $900{ }^{\circ} \mathrm{C}$, weak PL with the maximum at $650-750 \mathrm{~nm}$ is observed (Figure 6a). This light emission is usually assigned to different thermodynamically unstable radiative defects, for instance, nonbridging oxygen-hole centers or $E^{\prime}$-type defects [31,32,130-132]. After annealing above $900{ }^{\circ} \mathrm{C}$, the PL maximum shifts towards longer wavelengths $(\sim 800 \mathrm{~nm})$ and the PL intensity strongly increases [116,118], which agrees with numerous literature reports $[27,34,42,53,72,91]$. The highest PL intensity is observed for $x \cong 1.8-1.9$ and annealing at $1100-1150{ }^{\circ} \mathrm{C}$ [116]. Annealing at $1200{ }^{\circ} \mathrm{C}$ decreases the PL intensity. A similar decrease of the PL intensity was also observed for 
$\mathrm{SiO}_{x}(1.05<x<1.75)$ films prepared by magnetron sputtering [34]. A slightly different behavior is observed for $\mathrm{SiO}_{x}(1.05<x<1.75)$ films prepared by PECVD. For these samples, the PL intensity increases with the annealing temperature up to $1250{ }^{\circ} \mathrm{C}[34,40]$.
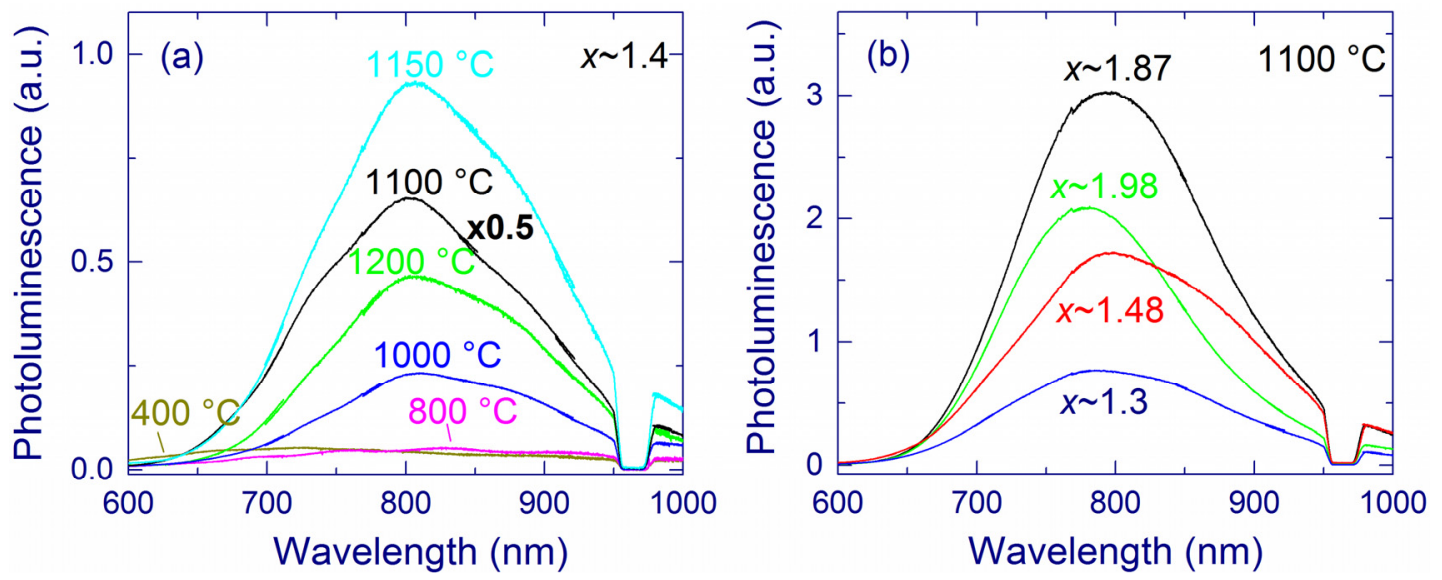

Figure 6. Photoluminescence (PL) spectra of (a) a $\mathrm{SiO}_{1.4}$ film annealed at different temperatures and (b) $\mathrm{SiO}_{x}$ films with different $x$ annealed at $1100{ }^{\circ} \mathrm{C}$. The $\mathrm{SiO}_{x}$ films are prepared by molecular beam deposition (MBD). The hole in the spectra around $970 \mathrm{~nm}$ is due to the second order of the notch filter. Notice the multiplication factor in (a). (b) is reproduced with permission from [116]. Copyright 2012, AIP Publishing LLC.

The enhancement of the $1.5-\mathrm{eV}$ PL by annealing at $\geq 1000{ }^{\circ} \mathrm{C}$ is accompanied by the formation of Si-nc detectable by Raman spectroscopy [40,48,49,116,118]. This correlation seemingly suggests that the PL directly originates from these Si-nc. On the other hand, the PL intensity is the highest for samples with relatively small Si contents $[48,49]$. Moreover, for $x>1.9$, no Si-nc and elemental Si are evidenced by the Raman and XPS measurements, respectively, whereas the PL is strong (Figure 6b). In fact, the PL quantum yield steadily increases as the absorption coefficient and the Si content decrease (Figure 7) [116,117]. These observations suggest that the Si-nc observed in the Raman spectra and detected by XPS as elemental Si are not direct light-emitting centers, which is in agreement with the defect origin of the 1.5-eV PL [52].

The PL position of our $\mathrm{SiO}_{x}$ films is rather stable for different annealing temperatures (above $1000{ }^{\circ} \mathrm{C}$ ) and $\mathrm{Si}$ contents (see Figure 6). These results seem to disagree with the studies of oxidized Si-nc prepared by magnetron sputtering [63]. Those experiments show that the PL position is size dependent for relatively large sizes $(>3 \mathrm{~nm})$ that are reliably measured by TEM. However, Wolkin et al. have shown that oxidation of Si-nc stabilizes the PL position for smaller Si-nc $(<3 \mathrm{~nm})$ [22].Thus, the stability of the PL position in our samples is consistent with the hypothesis that the observed PL originates from very small Si grains $(<3 \mathrm{~nm})$. In general, one has to distinguish different types of Si-nc samples. For example, the experiments on hydrogen-passivated Si-nc show that the PL of Si-nc with diameters smaller than $3 \mathrm{~nm}$ should be below $600 \mathrm{~nm}$ [23], which is not the case for our samples. This difference in PL positions can be explained by the decrease of the bandgap upon passivation of Si-nc with oxygen [110,133]. 




Figure 7. Relative PL quantum yield for $\mathrm{SiO}_{x}$ films prepared by MBD for annealing at $1000{ }^{\circ} \mathrm{C}$ (triangles), $1100{ }^{\circ} \mathrm{C}$ (squares), $1150{ }^{\circ} \mathrm{C}$ (diamonds), and $1200{ }^{\circ} \mathrm{C}$ (circles) as a function of the absorption coefficient at $488 \mathrm{~nm}$. The inset shows the relative PL quantum yield as a function of the amount of elemental Si for annealing at $1100{ }^{\circ} \mathrm{C}$. Reproduced with permission from [116]. Copyright 2012, AIP Publishing LLC.

Now we discuss the possibility of assigning the 1.5-eV PL to defects and/or ultra-small Si grains. By analogy with oxidized porous $\mathrm{Si}[22,62]$, the light-emitting centers can be related to the $\mathrm{Si}=\mathrm{O}$ covalent bonds $[27,48]$ or to the bridge $\mathrm{Si}-\mathrm{O}-\mathrm{Si}$ bonds suggested by theory [106]. Ultra-small (below $\sim 1 \mathrm{~nm}$ ) oxidized Si grains considered by theory [114] is another candidate to explain the red PL. Several observations are consistent with the latter interpretation. First, the stable PL position for different Si content suggests small sizes of the light-emitting clusters $(<3 \mathrm{~nm})$ [22]. The calculated band gaps of Si clusters with diameters $\sim 1 \mathrm{~nm}$ are $2-3 \mathrm{eV}$ [115], which agrees with the band gaps obtained for our samples (Figure 3) [116]. The short PL lifetimes obtained for our samples ( 1 $\mu$ s) [117] also suggest that the PL originates from Si-nc that are quite smaller than $3 \mathrm{~nm}$, for which the PL lifetime is $\sim 40 \mu \mathrm{s}[116,134]$. Moreover, the theoretical study by Luppi et al. suggests that Si-nc with

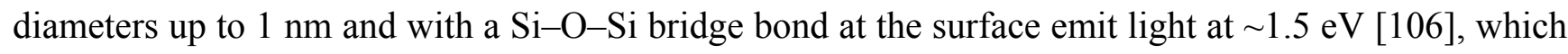
is observed for our samples. The decrease of the PL intensity after annealing at $1200{ }^{\circ} \mathrm{C}$ is reasonable if the amount of small optically active Si-nc decreases at this temperature.

The direct excitation of the light-emitting centers by laser light is possible. As another excitation mechanism, laser light may be absorbed by relatively large Si clusters, and then the excitation is transferred to the light-emitting centers located at the $\mathrm{Si}-\mathrm{nc} / \mathrm{SiO}_{2}$ interface $[68,135]$ or in more disordered areas $[48,117]$. Non-radiative defects such as dangling bonds at the $\mathrm{Si}-\mathrm{nc} / \mathrm{SiO}_{2}$ interface as well as defects inside and outside the Si-nc can efficiently trap the excitation and therefore quench the PL [53]. Annealing at $\sim 1100{ }^{\circ} \mathrm{C}$ presumably decreases the amount of these non-radiative defects and/or intensifies the energy transfer to the radiative centers, which enhances the PL. It has been shown that a considerable amount of non-paramagnetic near- and sub-gap defect states still remains after annealing, which drastically limits the PL quantum yield [136]. The amount of the light-emitting centers may increase upon annealing; however, no direct evidence of this is available.

Kusova et al. have suggested that compressive stress on $\mathrm{Si}-\mathrm{nc}$ in a $\mathrm{SiO}_{2}$ matrix can redshift the PL [137]. However, it is difficult to estimate stress of very small Si-nc using Raman spectroscopy, due to their low Raman scattering cross-section. Such small Si-nc can contribute to the broad low-frequency 
shoulder at $\sim 500 \mathrm{~cm}^{-1}$ (Figure 1). On the other hand, the Raman bands at $518-519 \mathrm{~cm}^{-1}$ observed in our samples feature unstressed Si-nc with sizes of $\sim 4 \mathrm{~nm}$ [121] that are also detected by TEM [125]. It can be speculated that no substantial stress is exerted also on smaller Si-nc.

\subsubsection{Comparison of the Implanted and $\mathrm{MBD} \mathrm{SiO}_{x}$ Samples $(x \sim 1.8)$}

After preparation, the structural and optical properties of the MBD and implanted samples are very different [118]. Amorphous Si clusters ( $>2 \mathrm{~nm}$ ) are present in the as-grown MBD film, as indicated by a broad Raman band at $\sim 40 \mathrm{~cm}^{-1}$ (Figure 1) and by a large absorption coefficient (Figure $8 \mathrm{a}$ ). For the implanted samples annealed below $900{ }^{\circ} \mathrm{C}$, the absence of Raman bands of Si and very low absorption (Figure 8a) suggest that only very small Si clusters (presumably with sizes up to $\sim 1 \mathrm{~nm}$ ) may be present in the material $[55,123]$.

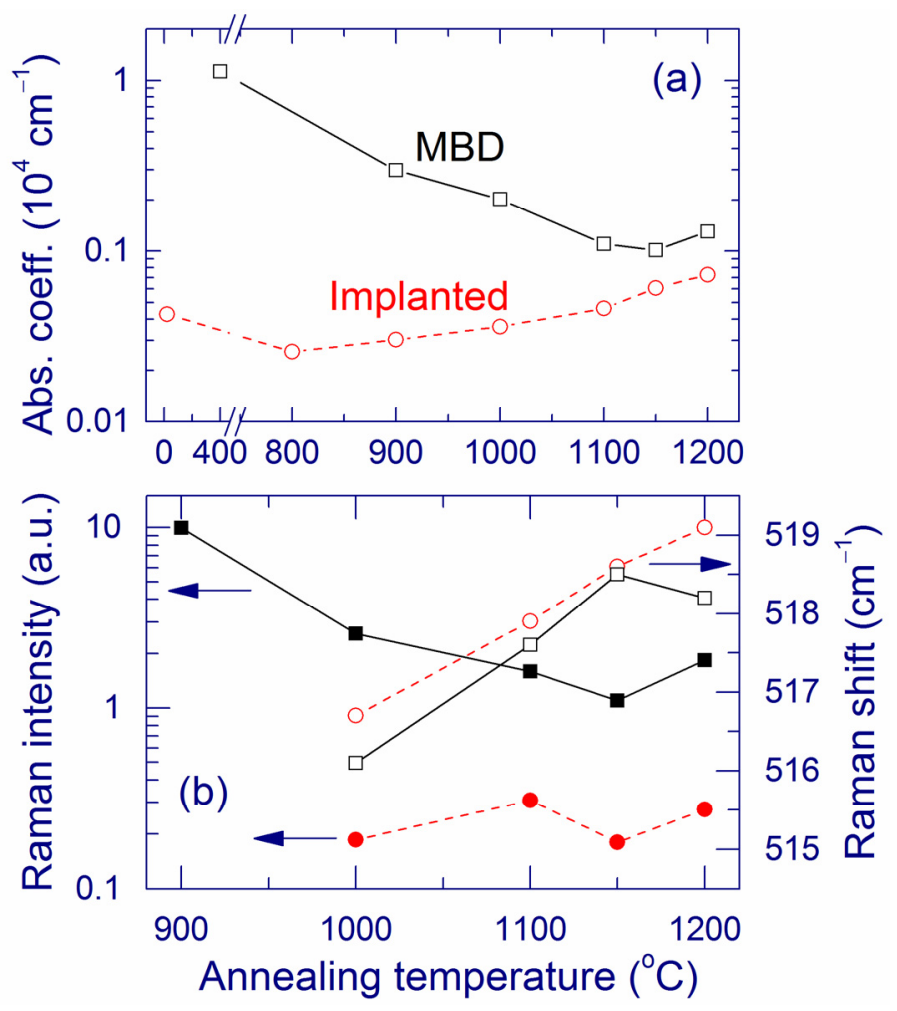

Figure 8. (a) Absorption coefficient at $488 \mathrm{~nm}$ for the MBD (squares) and implanted (circles) samples and (b) Normalized intensity (solid symbols) and position of the maximum (open symbols) of the Raman bands as a function of the annealing temperature. The Raman intensity is normalized by the effective film thickness (see Section 3.2). Reproduced with permission from [118]. Copyright Wiley-VCH Verlag GmbH \& Co. KGaA.

Upon annealing at $1000{ }^{\circ} \mathrm{C}$, a narrow Raman band at $\sim 516-517 \mathrm{~cm}^{-1}$ emerges for both types of samples, showing the formation of Si-nc. As compared to the as-prepared samples, the absorption coefficient of the annealed samples is increased for ion implantation but it is decreased for MBD (Figure 8a). After annealing at $1100-1200{ }^{\circ} \mathrm{C}$, both types of samples become optically and structurally more similar. For both types of samples, the Raman band is observed at $\sim 518 \mathrm{~cm}^{-1}$ and the absorption coefficients become closer (Figure 8). These trends are reasonably explained by growth and 
crystallization of Si clusters in the implanted sample and by crystallization of amorphous clusters in the MBD sample. Indeed, the extinction coefficient of crystalline $\mathrm{Si}$ is much smaller than that of amorphous $\mathrm{Si}$.

After annealing $\left(>1000{ }^{\circ} \mathrm{C}\right)$, the normalized Raman intensity and the absorption coefficient are larger for the MBD samples compared to the ion-implanted sample (Figure 8). This result indicates that the amount of Si-nc with diameters of $\geq 2 \mathrm{~nm}$ is greater in the MBD films. This conclusion is also supported by a smaller band gap for the MBD sample $(\sim 2.6 \mathrm{eV})$ compared to that for the implanted sample $(\sim 3.3 \mathrm{eV})$. For both types of samples, a substantial amount of suboxides $(\sim 15 \%)$ is detected by XPS even after annealing at $1200{ }^{\circ} \mathrm{C}$, indicating that the $\mathrm{Si}-\mathrm{SiO}_{2}$ phase separation is not complete.

For annealing below $900{ }^{\circ} \mathrm{C}$, the PL maximum is located at about 650-700 nm for both types of samples, and the PL intensity is much higher for the implanted sample (Figure 9). The latter fact indicates a larger amount of thermodynamically unstable radiative defects in the implanted sample [31,32,130-132]. Annealing at $\sim 1100^{\circ} \mathrm{C}$ enhances the 1.5-eV PL for both types of samples. After this annealing, the normalized PL is slightly stronger for the implanted sample (Figure 9), which is remarkable because the Raman band of Si-nc is weaker for this preparation method (Figure $8 b$ ). Annealing at $1200{ }^{\circ} \mathrm{C}$ weakens the PL, and this effect is more pronounced for the implanted sample. This decrease of the PL can originate from a thermal modification of the light-emitting centers, for example, an increase of the size of oxidized ultra-small Si grains. In the case of the implanted samples, this conclusion is supported by the redshift of the PL to $\sim 900 \mathrm{~nm}$ and by the decrease of the band gap from 3.3 to $2.8 \mathrm{eV}$ for annealing at 1150 and $1200{ }^{\circ} \mathrm{C}$, respectively.

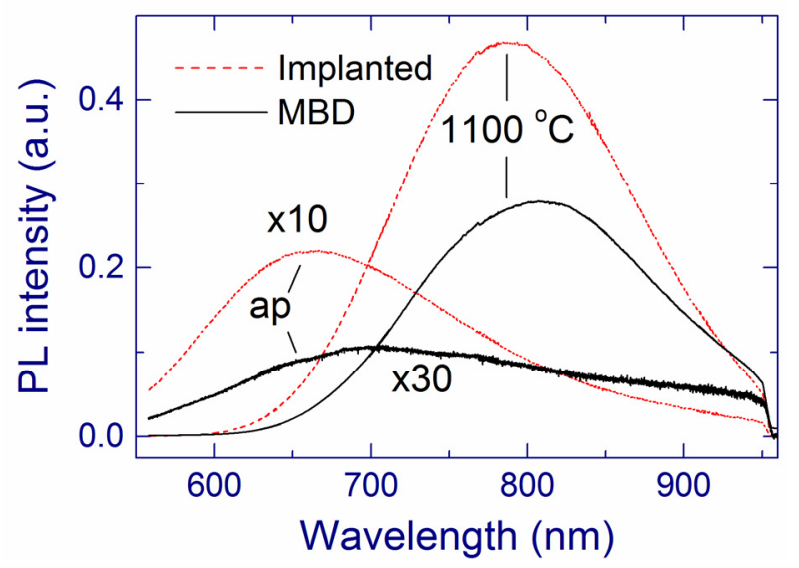

Figure 9. PL spectra of as-prepared (ap) and annealed $\left(1100^{\circ} \mathrm{C}\right) \mathrm{SiO}_{1.8}$ films fabricated by MBD and ion implantation. The PL spectra are normalized by the effective film thickness (see Section 3.2). Reproduced with permission from [118]. Copyright Wiley-VCH Verlag $\mathrm{GmbH} \& \mathrm{Co} . \mathrm{KGaA}$.

\subsubsection{Laser Heating of Films on Substrates}

Laser light is found to increase the temperature of $\mathrm{SiO}_{x}$ films on silica substrates [116]. For example, $488-\mathrm{nm}$ laser power of $\sim 130 \mathrm{~mW}$ focused to a $\sim 40-\mu \mathrm{m}$ spot (laser intensity $\sim 10^{4} \mathrm{~W} \mathrm{~cm}^{-2}$ ) leads to a temperature of $\sim 350{ }^{\circ} \mathrm{C}$ in a $\mathrm{SiO}_{1.3}$ film annealed at $1100{ }^{\circ} \mathrm{C}$. The absorption coefficient of this film increases at $350^{\circ} \mathrm{C}$ by $\sim 40 \%$ compared to the room temperature value, which is probably due 
to the thermally induced shrinking of the bang gap [97]. At this laser-induced temperature, the Raman band of Si-nc is observed at $\sim 512.5 \mathrm{~cm}^{-1}$, i.e., it is down-shifted by $\sim 6 \mathrm{~cm}^{-1}$ from the room temperature position. The temperature dependence of the Raman shift (Figure 10a) is in excellent agreement with the calculations by Faraci et al. for Si-nc with sizes of $5 \mathrm{~nm}$ [138]. Similar temperature dependences were previously reported for crystalline $\mathrm{Si}$ [139] and the free-standing $\mathrm{SiO}_{1.7}$ film [95]. The phonon confinement effect and residual stresses can explain the quantitative differences between the Raman band positions in these cases.
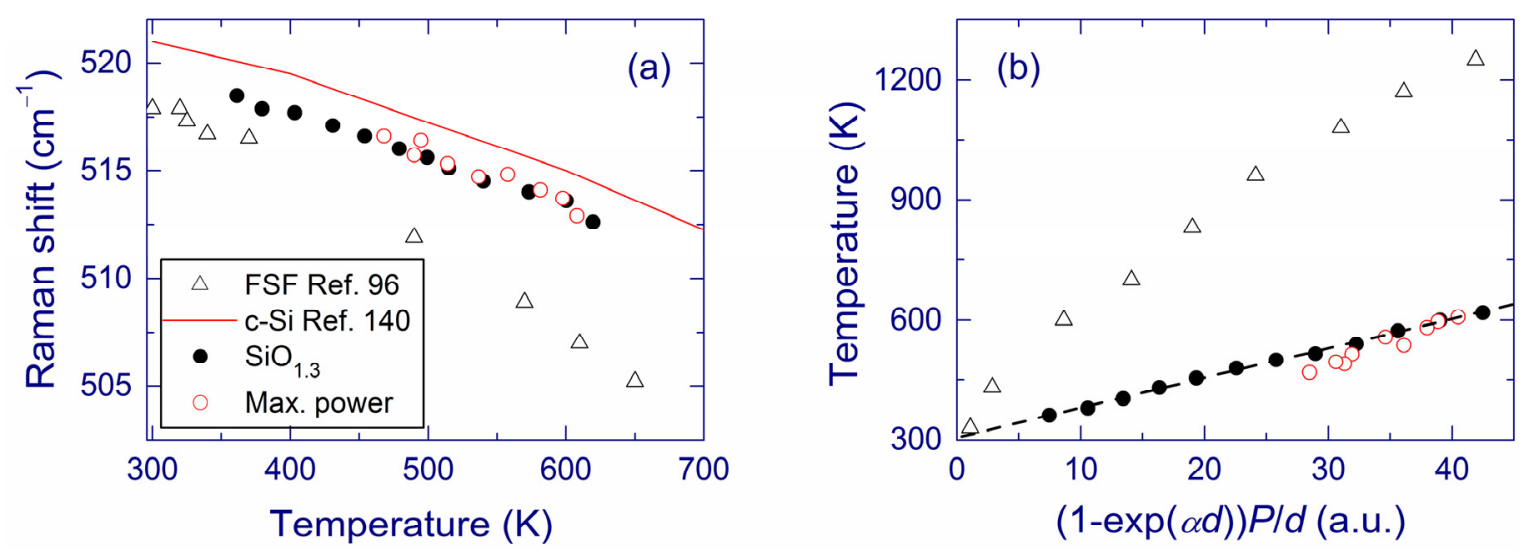

Figure 10. (a) Raman shift as a function of the laser-induced temperature and (b) Laser-induced temperature as a function of the absorbed laser power. The measurements are made for a $\mathrm{SiO}_{1.3}$ film on a silica substrate annealed at $1100{ }^{\circ} \mathrm{C}$ using various $488-\mathrm{nm}$ laser intensities (solid circles) and for $\mathrm{SiO}_{x}$ films $(1.3<x<1.5)$ at the maximum laser intensity of $\sim 10^{4} \mathrm{~W} \mathrm{~cm}^{-2}$ (open circles) [116]. The data for a free-standing $\mathrm{SiO}_{1.7}$ film [95] (FSF, triangles) and for crystalline Si [139] (solid line) are shown for comparison. The dashed line in panel $b$ is a linear fit. Reproduced with permission from [116]. Copyright 2012, AIP Publishing LLC.

Figure $10 \mathrm{~b}$ shows the laser-induced temperature for a free-standing $\mathrm{SiO}_{1.7}$ film and $\mathrm{SiO}_{x}$ films $(1.3 \leq x<1.5)$ on silica substrates as a function of the laser power absorbed by a unit volume, which is proportional to $\frac{\left(1-e^{\alpha d}\right) P}{d}$, where $P$ is the laser power, $\alpha$ is the absorption coefficient, and $d$ is the film thickness [95]. The linear fit of the temperature versus the absorbed power yields the room temperature at zero power, which verifies the results. For the same absorbed power, the laser-induced temperature is much higher in the free-standing films compared to the films on substrates, which is due to the thermal flow to the substrate in the latter case.

In Raman measurements of Si-nc, relatively high laser powers are usually employed to obtain a good signal-to-noise ratio. It follows that the laser-induced heat should always be carefully considered even for films on substrates (especially when using Raman microscopes) to obtain reliable information of the material structure. In general, laser heat, local stress, and phonon confinement are overlapping effects with respect to the Raman spectra. 


\subsection{Laser Annealing of Free-Standing Films}

\subsubsection{Laser-Annealed Areas}

We have studied CW laser annealing $(488 \mathrm{~nm})$ of free-standing $\mathrm{SiO}_{x}$ films $(0.5$ and $2 \mu \mathrm{m}$-thick) and $\mathrm{Si} / \mathrm{SiO}_{2} \mathrm{SL}(0.5-\mu \mathrm{m}$-thick). After preparation (Section 3.1), the free-standing films presumably contain small Si-nc (up to $4 \mathrm{~nm}$ in diameter) [95,125]. The laser annealing is usually performed for $\sim 1 \mathrm{~s}$ in air atmosphere (if different is not stated). An optical photograph of a typical laser-annealed area on a free-standing film and a Raman map from the same area are presented in Figure 11. Three regions can be distinguished: (i) central region, (ii) ring around the central region, and (iii) pristine film outside the irradiated area [140-142].
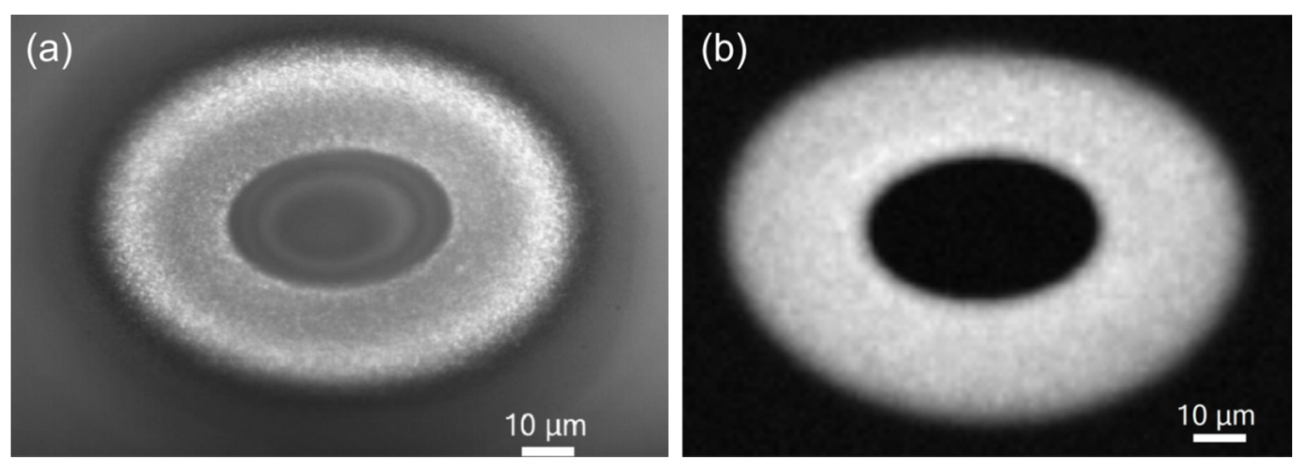

Figure 11. (a) Optical microscope photograph and (b) Raman map of the Si-nc band. A 2- $\mu$ m-thick free-standing $\mathrm{SiO}_{x}$ film $(x \sim 1.7-1.8)$ is exposed to $488-\mathrm{nm}$ light $(\sim 110 \mathrm{~mW})$ through a lens (focal length of $150 \mathrm{~mm}$ ). In the optical photograph, the sample is illuminated through the objective. The oval shape is due to a non-perpendicular direction of the annealing laser beam. Brighter regions in the Raman map correspond to stronger Raman signals. The Raman signal is integrated in the $510-540 \mathrm{~cm}^{-1}$ spectral range. Reproduced with permission from [140]. Copyright 2010, AIP Publishing LLC.

In the central region, the material is essentially amorphous $\mathrm{SiO}_{2}$. For 0.5 - $\mu$ m-thick $\mathrm{SiO}_{x}$ films $(x \sim 1.7-1.8)$, this composition is directly shown by XPS and electron energy loss spectroscopy (EELS) [140]. In accord, the TEM images and selected area diffraction (SAD) patterns indicate a very homogeneous and amorphous structure of this region, and the Fourier-transform infrared spectra (FTIR) measured with high spatial resolution are similar to those of thermal silica. In this region, no Si-related peak is detected by Raman spectroscopy, the absorption is very low, and no PL is observed (Figure 12). In agreement, for $0.5-\mu$ m-thick $\mathrm{Si} / \mathrm{SiO}_{2} \mathrm{SL}$ (2-nm-thick $\mathrm{Si}$ and $\mathrm{SiO}_{2}$ layers), no $\mathrm{Si}$ clusters are detected in bright-field scanning TEM (BF-STEM) images, and energy-dispersive X-ray spectroscopy (EDS) indicates an increase of $x$ in the central region compared to the pristine film [141,142]. Quantitative analysis based on the EDS measurements is not possible because quantification using low-energy $\mathrm{X}$-ray lines such as oxygen $\mathrm{K}$ alpha is not feasible. Small Si clusters $(<1 \mathrm{~nm})$ invisible by these microscopic methods are in principle possible in the central region. However, very small Si clusters are thermodynamically unfavorable [143], and their formation seems to be improbable at high temperatures. Furthermore, these very small Si grains are presumably luminescent; however, practically no PL is detected from the central region. 

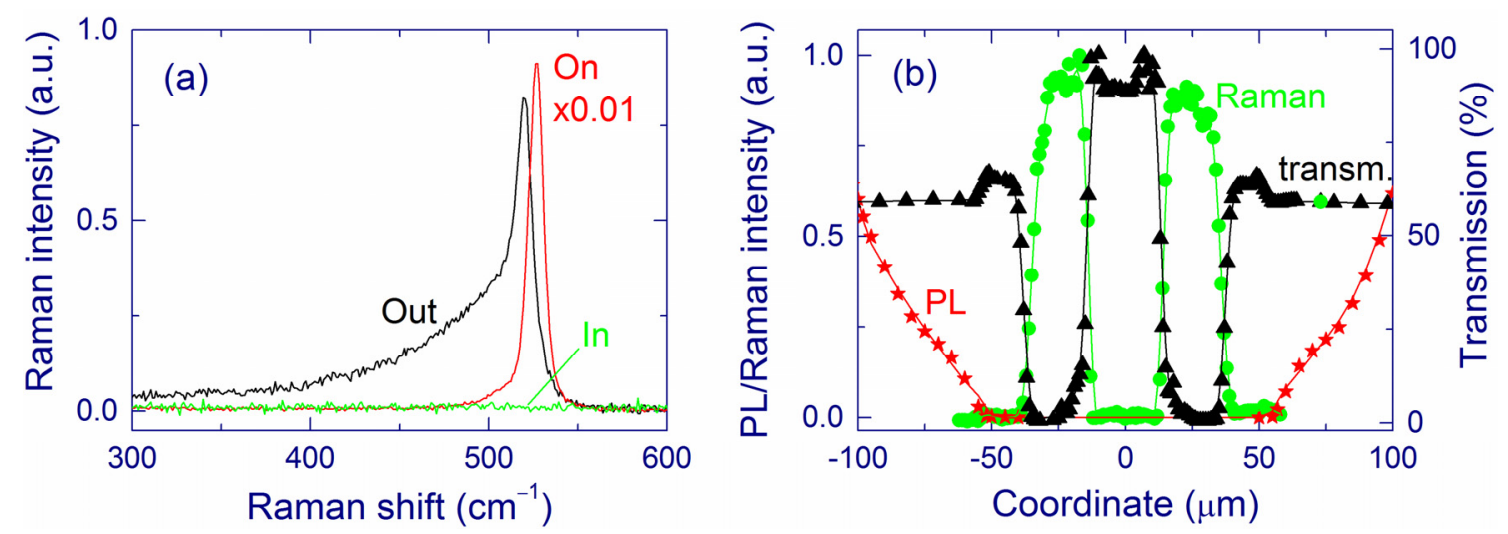

Figure 12. (a) Raman spectra of a laser-annealed 2- $\mu$ m-thick $\mathrm{SiO}_{x}(x \sim 1.7-1.8)$ film from regions in the center (in), on the ring (on), and outside the laser-annealed area (out). (b) Raman (circles), PL (stars), and transmission (triangles) cross-sections of the laser-annealed area. The cross-sections are measured in the vertical direction through the center of the laser-annealed area shown in Figure 11. Reproduced with permission from [140]. Copyright 2010, AIP Publishing LLC.

In the ring around the central region, the EFTEM, BF-STEM, and TEM images evidence large $\mathrm{Si}$-nc embedded in amorphous $\mathrm{SiO}_{2}$ (Figure 13) [140-142]. The maximum Si-nc diameters $(\sim 200 \mathrm{~nm})$ for the $0.5-\mu \mathrm{m}$-thick $\mathrm{Si} / \mathrm{SiO}_{2} \mathrm{SLs}$ [141] are on average two times larger than those detected for the $0.5-\mu \mathrm{m}$-thick free-standing $\mathrm{SiO}_{x}$ films $(x \sim 1.7-1.8)$ [140]. This difference is reasonably explained by a larger $\mathrm{Si}$ content in $\mathrm{Si} / \mathrm{SiO}_{2} \mathrm{SLs}(x \sim 0.7$ from the deposition conditions) compared to the $\mathrm{SiO}_{x}$ films $(x \sim 1.7-1.8)$. The crystallinity of the large $\mathrm{Si}$ clusters in the ring region is confirmed by selected-area diffraction measurements (for 0.5 - $\mu$ m-thick $\mathrm{SiO}_{x}$ film) and Raman spectroscopy. The Raman band is quite narrow and does not have a shoulder at $450-500 \mathrm{~cm}^{-1}$, indicating the absence of amorphous phase and therefore suggesting efficient Si crystallization (see Figure 12a). The EELS spectra show the presence of the $\mathrm{Si}$ and $\mathrm{SiO}_{2}$ phases. In accord, the FTIR spectra correspond to stoichiometric $\mathrm{SiO}_{2}$ perturbed by the $\mathrm{Si}-\mathrm{SiO}_{2}$ interface. The Raman bands from the ring region is stronger by about 2-3 orders of magnitude compared to the pristine film, which is explained by the resonance size effect for the large Si-nc [144]. The Raman bands from Si-nc in the ring region are typically shifted up to $\sim 530 \mathrm{~cm}^{-1}$ (from the value of $521 \mathrm{~cm}^{-1}$ for crystalline $\mathrm{Si}$ ) indicating a compressive stress on Si-nc. According to the mechanism proposed earlier [56], these large Si-nc are formed from the liquid phase, and the stress appears when their volume suddenly increased in the solid $\mathrm{SiO}_{2}$ matrix. Thus, the temperature in the ring region exceeds the melting point of Si $(1685 \mathrm{~K})$. Indeed, a temperature of $\sim 2000 \mathrm{~K}$ was estimated by fitting the strong light-emission background upon irradiation with high laser intensities ( $\sim 16 \mathrm{~kW} \mathrm{~cm}^{-2}$ at the sample) [94]. The bright thermal radiation is also seen by the naked eye. High processing temperatures in the central region are also confirmed by the decrease of the film roughness detected by AFM. For $0.5-\mu \mathrm{m}$-thick $\mathrm{Si} / \mathrm{SiO}_{2} \mathrm{SLs}$, scanning electron microscopy (SEM), TEM, and atomic force microscopy (AFM) show several large holes (up to $200 \mathrm{~nm}$ ) in the inner part of the ring region (Figure 13a,b).

These results demonstrate a $\mathrm{Si}-\mathrm{SiO}_{2}$ phase separation on a macroscopic scale. The central region (up to $30 \mu \mathrm{m}$ ) is cleaned of the Si excess. On the other hand, in the ring region, large Si-nc are formed 
and the $\mathrm{Si}$ excess is enhanced as found for $0.5-\mu \mathrm{m}$-thick $\mathrm{SiO}_{x}$ film by XPS and EELS [140]. The macroscopic phase separation can be explained as follows [140]. In the beginning of laser irradiation, the film structure is homogeneous so that the temperature gradient approximately corresponds to the intensity gradient in the Gaussian beam. The temperature gradient gives rise to a concentration gradient due to different diffusion properties of the mixture constituents. For example, $\mathrm{Si}, \mathrm{O}$, and $\mathrm{SiO}$ can diffuse in silica [145], which changes the relative concentrations. Diffusion in temperature gradient is known as thermodiffusion or Ludwig-Soret effect [146,147]. As detected by EELS, XPS, and AFM, the central region is typically thinner than the pristine film by about $30 \%-40 \%$ and $\sim 50 \%$ for $0.5-\mu \mathrm{m}$-thick $\mathrm{Si} / \mathrm{SiO}_{2} \mathrm{SLs}$ and $\mathrm{SiO}_{x}$ films, respectively. Moreover, AFM shows an increase of the film thickness in the ring region compared to the pristine film [142]. These results are also indicative of macroscopic movement of Si excess from the central region.
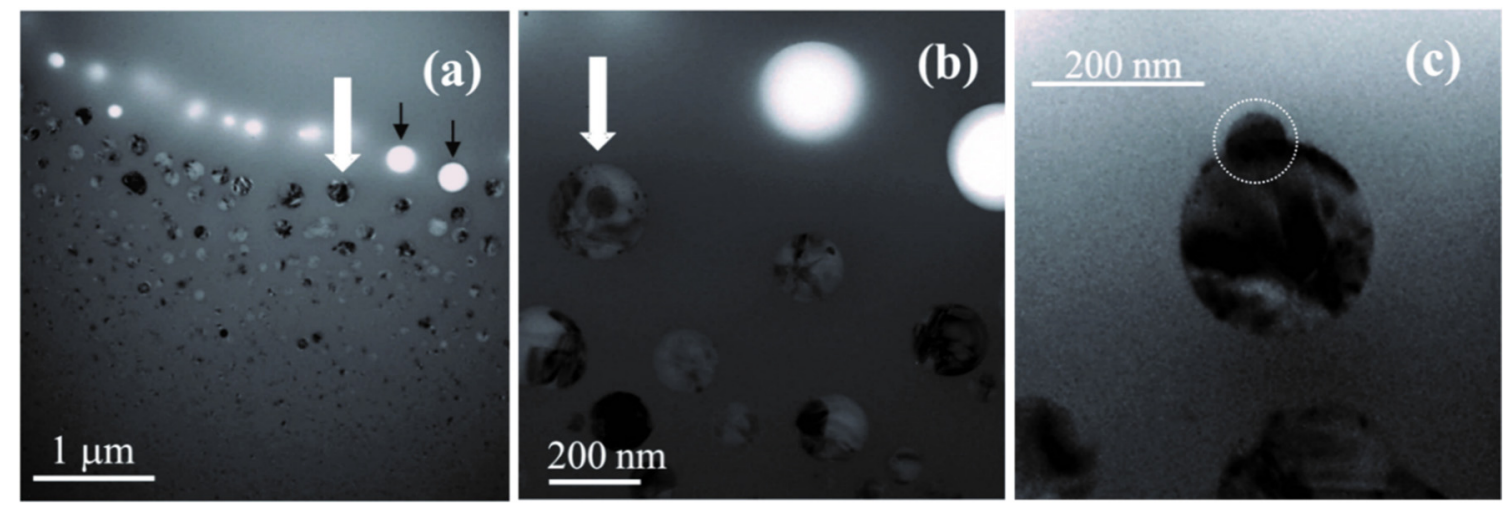

Figure 13. TEM images of a $0.5-\mu \mathrm{m}$-thick $\mathrm{Si} / \mathrm{SiO}_{2} \mathrm{SL}$ showing a $\mathrm{Si}$ particle located near the film surface (marked with a white arrow). The images in (a), (b), and (c) are taken with different magnification. (c) shows this particle in the tilt geometry, the surface subparticle being indicated by a dotted circle. The bright spots are holes in the film; the holes observed in (b) are marked by black arrows in (a). Figure courtesy of Simona Boninelli and Fabio Iacona. Reproduced with permission from [141]. Copyright 2012, AIP Publishing LLC.

\subsubsection{Surface Structure}

For laser-annealed $0.5-\mu \mathrm{m}$-thick $\mathrm{Si} / \mathrm{SiO}_{2} \mathrm{SLs}$, surface analysis (SEM and AFM) has been used to discriminate Si-nc located near the film surface [141]. In the tilt TEM images (Figure 13c), these "surface Si-nc" have an unusual pear-like shape with the thinner part presumably sticking out of the laser-illuminated surface. The AFM and SEM methods estimate that the thinner part above the surface (surface feature) has a typical size of $60-70 \mathrm{~nm}$, in agreement with the TEM measurements. The subsurface part has a diameter of $200 \mathrm{~nm}$, as shown by TEM and BF-STEM images.

The formation of the surface features is explained by the following mechanism. Since most of large Si-nc formed by laser annealing are under high compressive stress, it is possible that the laser-pressurized silicon can erupt from the film if it is separated from the ambient atmosphere by a very thin silica layer. The erupted $\mathrm{Si}$ is seen as the subparticle in Figure 13c. The volume of the subparticle ( $\sim 70 \mathrm{~nm}$ in diameter) is $\sim 5 \%$ of the volume of the larger subsurface part of the nanocrystal ( $\sim 200 \mathrm{~nm}$ in diameter). Eruption of this volume would lead to a substantial decrease of the compressive 
stress of the parent particle [56]. The fact that no eruptions are detected on the backside of the film seems to be consistent with this mechanism. Indeed, the temperature should be somewhat higher on the front (laser-illuminated) side of the film; hence, the covering silica film is softer here, which favours Si eruption.

Raman measurements confirm the proposed eruption model. The surface Si nanocrystal shown in the TEM images in Figure 13 is located in position 1 in Figure 14a. The Raman shift obtained in this position is $\sim 520 \mathrm{~cm}^{-1}$ (spectrum 1, Figure 14b), which indicates a low stress. It is reasonable that $\mathrm{Si}$ eruption can relax stress in the vicinity of surface Si-nc. The compressive stress is partially relaxed at a distance of about $1 \mu \mathrm{m}$ from the surface feature (spectrum 2). The Raman spectrum measured at position 3, which is far away from the surface feature, shows a higher compressive stress (spectrum 3 in Figure 14b).


Figure 14. Raman characterization of the area shown in Figure 13a. (a) Raman map of Si-nc (integration from 515 to $530 \mathrm{~cm}^{-1}$ ) obtained with a $100 \times$ objective and steps of $0.2 \mu \mathrm{m}$. Brighter areas correspond to stronger Raman signals. The white circles indicate the positions of two holes used as the reference points. (b) Raman spectra in positions 1, 2, and 3 indicated in (a). Position 1 corresponds to the surface Si-nc shown in Figure 13. Reproduced with permission from [141]. Copyright 2012, AIP Publishing LLC.

\subsubsection{Effect of the Annealing Atmosphere}

The studies described above have been performed in the atmosphere of air (containing $\sim 20 \%$ of oxygen), which can cause some oxidation of Si during laser annealing. Indeed, oxygen can diffuse through the film oxide to Si-nc [148-150]. This process may decrease the excess of Si, especially in the central region, where the temperature in the beginning of laser annealing is the highest. To evaluate this effect, we compare laser annealing in atmospheres containing different amounts of oxygen (Ar, $\mathrm{N}_{2}, \mathrm{O}_{2}$, and air). Detailed measurements are performed for $0.5-\mu \mathrm{m}$-thick $\mathrm{Si} / \mathrm{SiO}_{2} \mathrm{SL}$ and 1 -s exposure [142].

For all annealing atmospheres, the central region of laser-annealed areas most probably consists of amorphous silica without Si excess. This composition is indicated by the absence of the Raman signal of $\mathrm{Si}$ in this region, an increase of $x$ compared to the pristine film detected by EDS, and the absence of Si clusters in the BF-STEM images. In the ring region, large Si-nc (up to 150-300 nm) are formed, and the film thickness tends to increase compared to that of the pristine film. The change of the film 
thickness was estimated using the AFM images taken from both sides of the film. These results support the concept of macroscopic $\mathrm{Si}-\mathrm{SiO}_{2}$ phase separation as a result of laser annealing.

The effect of oxygen can be analyzed by comparing the film thickness in the central region obtained for different atmospheres. Indeed, two competitive processes, thermodiffusion and Si oxidation, affect the film thickness in the opposite directions, tending, respectively, to decrease and to increase it. For Ar atmospheres, the central region is thinner by $\sim 150 \mathrm{~nm}$ than the pristine film, i.e., the thickness is about $350 \mathrm{~nm}$. This observation supports the concept of the macroscopic phase separation because $\mathrm{Si}$ oxidation does not operate in the inert atmospheres. A similar change of the thickness occurs in air (the thickness of the central region is $300-350 \mathrm{~nm}$ ) also indicating minor Si oxidation.

In contrast, laser annealing in pure $\mathrm{O}_{2}$ increases the film thickness in the central region by $\sim 20 \mathrm{~nm}$ compared to the pristine film. Thus, both thermodiffusion and Si oxidation seem to operate in this case, practically compensating each other. It seems that a significant part of Si is oxidized upon laser annealing in pure oxygen. The difference between the film thickness of the central region for inert and $\mathrm{O}_{2}$ atmospheres is $\sim 200 \mathrm{~nm}$ (larger in oxygen). $200 \mathrm{~nm}$ of $\mathrm{SiO}_{2}$ can be formed by oxidation of about $100-\mathrm{nm}$ Si layer, which corresponds to $\sim 40 \%$ of all amount of elemental Si in the pristine SL (125 2-nm-thick Si layer). According to the model suggested by Deal and Grove for crystalline Si, 1 bar of dry $\mathrm{O}_{2}$ at $1200{ }^{\circ} \mathrm{C}$ produces $\sim 20$-nm oxide film during $1 \mathrm{~s}$, whereas a $\sim 33-\mathrm{nm}$ oxide is formed for $100 \mathrm{~s}$ [149], which is in approximate agreement with our estimates. It should be reminded that the temperature of laser annealing is sufficiently higher [94]. In addition, the extent of oxidation can differ from these values because our pristine material is very different from crystalline Si.

The structure of the ring region is also affected by the amount of $\mathrm{O}_{2}$ in the ambient atmosphere. For inert atmospheres, the AFM and BF-STEM images show that the ring region is very inhomogeneous. It has areas with large Si-nc (up to $150 \mathrm{~nm}$ in diameter), with large non-spherical holes up to $300 \mathrm{~nm}$, and with smaller Si-nc (up to $50 \mathrm{~nm}$ in diameter). No surface Si-nc are observed in this case, as shown by AFM and SEM. Raman spectroscopy indicates Si-nc with different stresses (Raman shifts from 518 to $530 \mathrm{~cm}^{-1}$ ) and regions with very different intensities of Raman bands of Si-nc. The BF-STEM images show a "belt" of nano-channels (or pores) around the ring region. The size of these nano-channels can be as low as several nanometers.

For laser annealing in air, the ring region contains Si-nc with diameters of up to $200 \mu \mathrm{m}$. A chain of holes with sizes up to $200 \mu \mathrm{m}$ is seen at the inner part of the ring of large Si-nc (Figure 15a). A number of surface features are observed as discussed above.

For pure $\mathrm{O}_{2}$ atmosphere, in addition to Si-nc inside the film, SEM and AFM show a regular chain of surface features that are located in the inner part of the ring at the front side of the film (Figure 15b). The undersurface part of the surface Si-nc can be up to $300 \mathrm{~nm}$ in diameter. In agreement with the suggested eruption model, Raman spectroscopy shows a relaxed stress for these surface Si-nc (Raman shift of $\sim 520 \mathrm{~cm}^{-1}$ ) whereas the Si-nc at the outer part are still under compressive stress (Raman shift up to $\sim 530 \mathrm{~cm}^{-1}$ ). No holes in the film are observed after laser annealing in $\mathrm{O}_{2}$ atmosphere. 

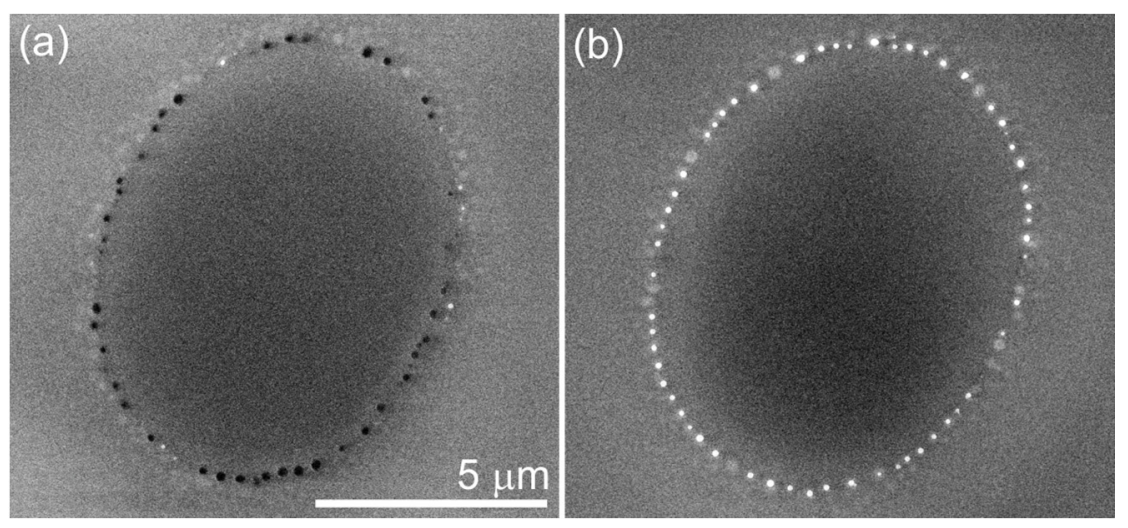

Figure 15. Secondary electron (SE) images of the front side of laser-annealed areas produced in (a) air and (b) $\mathrm{O}_{2}$ atmospheres during 1-s exposure. Surface features are white, holes are black. The scale is the same in both panels. Figure courtesy of Marianna Kemell. Reprinted with permission from [142]. Copyright 2014, American Scientific Publishers.

Based on these results, it can be suggested that the holes produced in inert and air atmospheres are formed via removal of surface Si-nc from the film. For laser annealing in air, the holes appear in the positions where a chain of large surface Si-nc is observed in the case of $\mathrm{O}_{2}$ atmosphere (see Figure 15). The laser-annealed areas with larger number of holes contain less surface Si-nc, and the presence of oxygen increases the amount of surface Si-nc. This observation suggests that oxygen decreases the removal of large Si-nc by the formation of a thin oxide layer covering surface Si-nc, which "glues" them to the film. The large irregular holes obtained in the inert atmospheres also presumably originate from large Si-nc removed from the film. The suggested mechanism of hole formation is consistent with the dependence of the maximum Si-nc sizes on the annealing atmosphere. For inert atmospheres, the largest Si-nc visible in the BF-STEM images are "only" 150 nm in diameter because bigger Si-nc are removed from the films forming holes with sizes of up to $300 \mathrm{~nm}$. For $\mathrm{O}_{2}$ atmosphere, the largest Si-nc (up to $300 \mathrm{~nm}$ ) are detected because they are protected by thin oxide layers. Annealing in air represents an intermediate case where the largest Si-nc are $200 \mathrm{~nm}$ in diameter, and some Si-nc are removed forming holes. In accord, with the proposed mechanism, holes are not observed in a 0.5- $\mu$ m-thick $\mathrm{SiO}_{x}$ film $(x \sim 1.7-1.8)$ annealed in air [140]. For this film, Si-nc have diameters "only" up to $100 \mathrm{~nm}$, which are substantially smaller than the film thickness. These Si-nc are mostly located below the film surface and, hence cannot be removed to form holes.

Figure 16 shows the cross-sections of the Raman signal measured through characteristic ring regions of the laser-annealed areas prepared in inert (Ar) and $\mathrm{O}_{2}$ atmospheres. For inhomogeneous ring regions formed in Ar atmosphere, two different characteristic cross-sections are observed (Figure 16a,b) whereas for $\mathrm{O}_{2}$ and air atmospheres, the laser-annealed area is more uniform having similar Raman cross-sections in different ring regions (see Figure $16 \mathrm{c}$ for $\mathrm{O}_{2}$ ). For 488-nm laser light, the Raman enhancement is known to be the strongest for spherical Si particles with diameters of $\sim 112.5 \mathrm{~nm}$ [144]. In agreement, Si-nc with sizes $\sim 100 \mathrm{~nm}$ are observed in the areas with the strongest Raman signal (at coordinate $=0$ ). At coordinates $>5 \mu \mathrm{m}$, the Raman spectra are similar to that of the pristine film. In Figure 16b, the Raman intensity is relatively weak in the ring region because the Si-nc diameters in this case do not exceed $\sim 50 \mathrm{~nm}$, which is smaller than the resonance value of $\sim 112.5 \mathrm{~nm}$. 
For inert atmospheres (Figure 16a,b), the Raman signal is very weak at coordinates $2-3 \mu \mathrm{m}$. For air and $\mathrm{O}_{2}$, this decrease of Raman intensity is less pronounced but still observable (Figure 16c). We call this effect the secondary Si depletion, and it supports the model of macroscopic phase separation. We tentatively explain the formation of this interval with low Raman intensity in the following way. When the large Si-nc are formed in the ring region during laser annealing, the absorption coefficient in this area strongly increases resulting in an increase of the temperature. Due to the thermal conductivity, the temperature of the vicinity also increases, which leads to a temperature gradient and additional macroscopic phase separation outside the ring region. This process seems to be more efficient for the inert compared to oxygen-containing atmospheres. The position of this spatial interval roughly coincides with the position of the belt of nano-pores and nano-channels observed by SEM in the inert atmospheres. The exact mechanism of the formation of these pores is unclear.

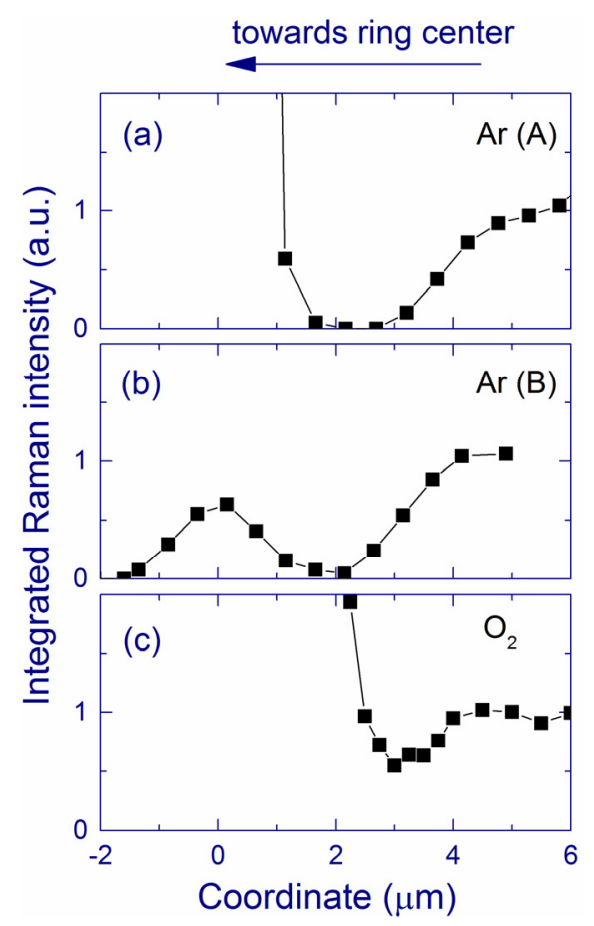

Figure 16. Characteristic Raman cross-sections of the ring region. Two characteristic cross-sections (a) and (b) are shown for Ar atmosphere, and the cross-sections (c) are similar for $\mathrm{O}_{2}$. For air, the cross-sections are similar to the case of $\mathrm{O}_{2}$. The Raman signal is integrated in the $230-585 \mathrm{~cm}^{-1}$ range. The data are normalized by the Raman intensity measured from the pristine film. Reprinted with permission from [142]. Copyright 2014, American Scientific Publishers.

\subsubsection{Effect of the Exposure Period}

Laser annealing with different exposures $(10 \mathrm{~ms}$ and $100 \mathrm{~s}$, in addition to $1 \mathrm{~s})$ has been studied for $0.5-\mu \mathrm{m}$-thick $\mathrm{Si} / \mathrm{SiO}_{2} \mathrm{SL}$ mostly in air atmosphere [142]. For the central area, the results are similar for all exposures: the EDS measurements show an increase of $x$ (relative to the pristine film), no Raman signal from Si-nc is detected, and no Si clusters are visible in the BF-STEM images. Thus, 10-ms exposure is enough for the removal of excess Si from the central region. In other words, the formation of the central region occurs in a millisecond time scale. 
On the other hand, the ring region differs for different exposures. For 10-ms exposures, no holes and surface features are found and Si-nc have diameters of up to 20-30 nm. For 1-s and 100-s laser exposures, some holes and surface features are formed and Si-nc with diameters up to 200 and $250 \mathrm{~nm}$, respectively, are observed. Thus, longer laser exposure increases the Si-nc size. Coalescence of Si-nc can be suggested for this process [73], because the number of Si-nc decreases for longer exposure periods. The increase of the Si-nc sizes with the exposure period can probably explain the greater amount of surface Si-nc observed for 100-s compared to 1-s exposure. For other atmospheres, the prolonged laser annealing (100 s) also decreases the amount of small Si-nc and increases the average sizes of Si-nc compared to 1-s exposure.

The Si-nc diameters for 10-ms exposure (20-30 nm) are smaller than the resonance size of $112.5 \mathrm{~nm}$. This explains why no Raman enhancement is observed from these Si-nc (Figure 17). In contrast, for 1-s and 100-s exposures, the Raman intensity is strongly enhanced compared to that from the pristine film, which is explained by the presence of Si-nc with diameters of $\sim 100 \mathrm{~nm}$. The Raman band observed after 10-ms exposure has a smaller low-frequency shoulder compared to the pristine film, meaning that the amount of disordered $\mathrm{Si}$ and/or small $\mathrm{Si}$ clusters is reduced. As the exposure increases, the Raman band narrows indicating that the structure of Si-nc becomes more regular. For 100-s exposure, the width of the Si-nc Raman band is as narrow as that of a crystalline Si wafer, which indicates an almost perfect crystalline structure.

For 10-ms and 1-s exposures, the Si-nc in the ring region are under compressive stress because the Raman shifts are larger than that of crystalline Si (Figure 17); thus, these Si-nc are formed from the liquid phase. On the contrary, no stress on Si-nc is detected for 100-s exposure for all studied annealing atmospheres. This fact may show that for the long exposure, the temperature of large Si-nc becomes at some moment below the melting point. The decrease of the temperature for long exposure may be explained by the decrease of the absorption when the Si-nc become much larger than the resonance size of $112.5 \mathrm{~nm}$ [144]. Annealing at temperatures below the melting point relaxes the stress [56,96].

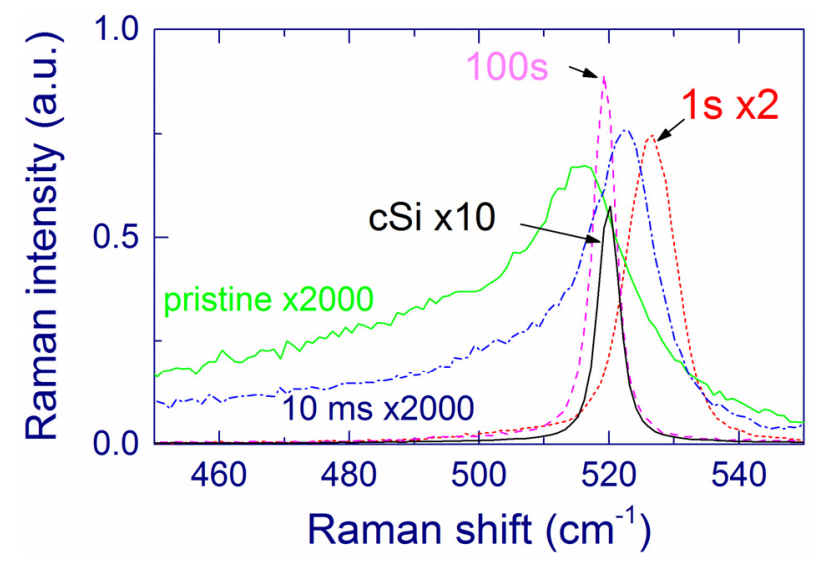

Figure 17. Typical Raman spectra of the ring region for 10-ms, 1-s, and 100-s exposures in air. Raman spectra of the pristine film and crystalline $\mathrm{Si}$ wafer (cSi) are also shown. Reprinted with permission from [142]. Copyright 2014, American Scientific Publishers. 


\subsubsection{Optical Memory}

We describe two concepts of optical memory based on laser annealing of Si nanostructures in free-standing $\mathrm{Si} / \mathrm{SiO}_{2} \mathrm{SLs}$ [151]. The first concept is based on the enhancement of Raman signal. The sample is irradiated by a $488 \mathrm{~nm}$ laser beam focused by a $100 \times$ objective of a Raman microscope, which produces laser-annealed areas with sizes below $1 \mu \mathrm{m}$. These areas are clearly visible in camera photographs (Figure 18a), and this indicates a change in the reflection and/or absorption coefficient of the film. The Raman, PL, and transmission profiles through a typical laser-annealed spot are presented in Figure 18b. The Raman signal is strongly enhanced whereas the transmission is decreased due to temperature-induced $\mathrm{Si}-\mathrm{SiO}_{2}$ phase separation and ordering and growth of $\mathrm{Si}-\mathrm{nc}$ [140-142].

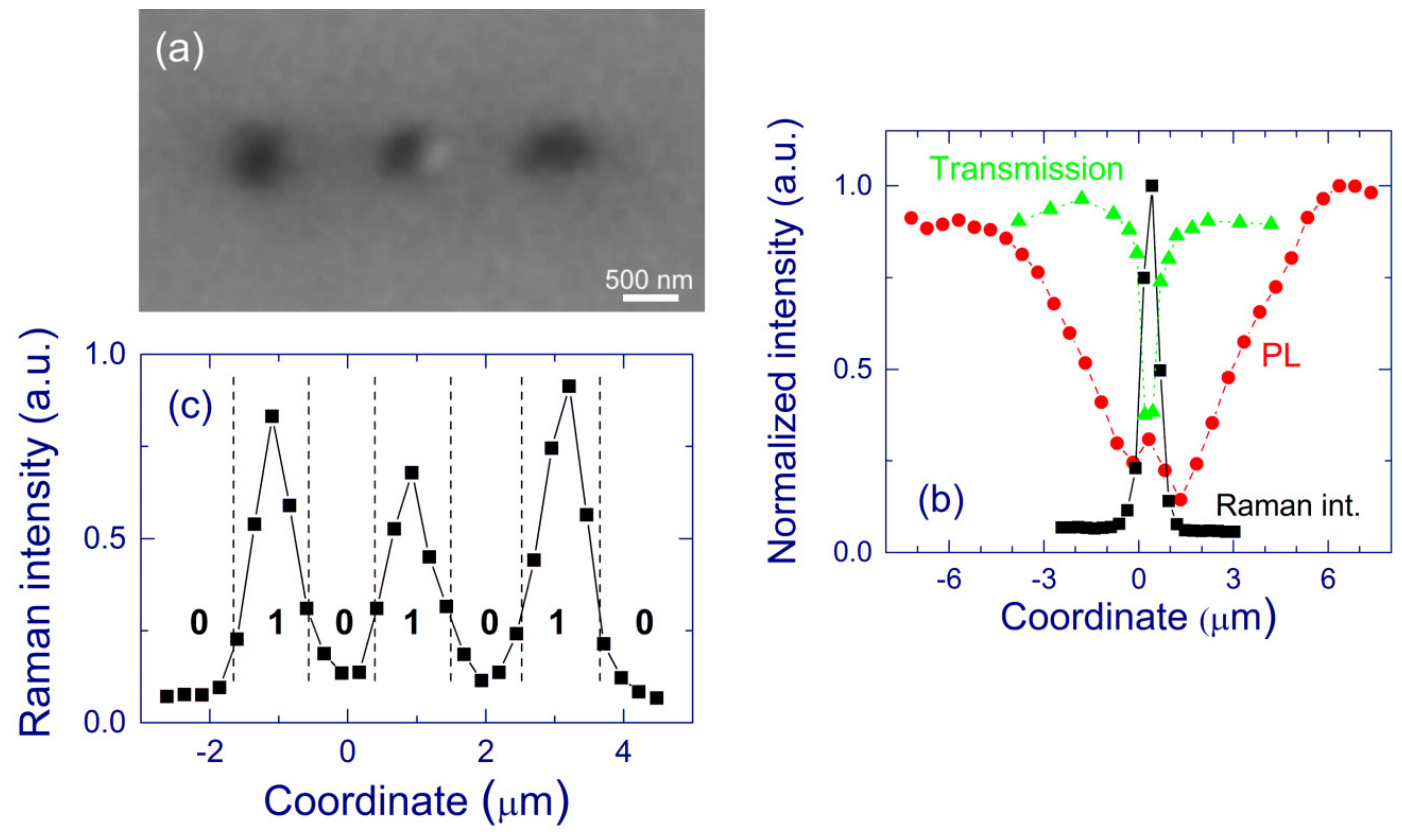

Figure 18. Writing digital data on a free-standing 2- $\mu$ m-thick $\mathrm{Si} / \mathrm{SiO}_{2} \mathrm{SL}$. (a) Camera photograph of three spots produced by 488-nm laser annealing for $\sim 10 \mathrm{~ms}$. (b) Cross-section of the Raman, transmission, and 1.5-eV PL signals through a typical laser spot. (c) Cross-section of the Raman intensity through the spots presented in panel a, which illustrates digital data points. The Raman intensity is integrated in the $500-540 \mathrm{~cm}^{-1}$ range. Reproduced with permission from [151]. Copyright 2009, AIP Publishing LLC.

The observed laser effect is a case of non-volatile memory. Binary digits can take values of either 0 or 1 as demonstrated in Figure 18c. The smaller Raman intensity corresponds to 0, whereas the higher Raman intensity represents 1 . Similar data reading can be obtained by transmission or reflection measurements. The estimated data density for this method is $\sim 1 \mathrm{bit} / \mu \mathrm{m}^{2}$, which can be improved by using stronger objectives and shorter laser wavelength. The nature of the used material provides high thermal stability of the described optical memory. The thermal stability of the laser-crystallized spots was tested by furnace annealing for $1 \mathrm{~h}$ at $1200{ }^{\circ} \mathrm{C}$ in a nitrogen atmosphere. As expected, Raman and transmission signals remained unchanged. This approach does not allow erasing and rewriting information. 
The second concept is based on Si-nc stress. First, a free-standing $\mathrm{Si} / \mathrm{SiO}_{2} \mathrm{SL}$ is shortly irradiated with a relatively large laser spot (10-40 $\mu \mathrm{m}$ in diameter), which produces an extended area with a compressive stress of $\sim 3 \mathrm{GPa}$ (see Figure 11). This stress is evidenced by Raman bands at $\sim 527 \mathrm{~cm}^{-1}$ (Figure 19a). Next, the stressed area is irradiated by a highly focused laser beam using a $100 \times$ objective and reduced laser power. As a result of laser annealing below the Si melting temperature, the stress is released in the irradiated spot and the Raman band shifts down to $\sim 518 \mathrm{~cm}^{-1}$. The typical size of the spot with relaxed stress is $\sim 1 \mu \mathrm{m}$ (Figure 19b). The optical constants are practically unchanged upon the stress relaxation, and this effect is invisible in optical photographs. The laser-induced decrease of the Raman shift writes data pixels on the film similarly to the concept based on the Raman enhancement. The data can be erased by inducing stress using a broad laser beam and then re-written again. The retention time of the stress-based memory is estimated to be about 1 year at $\sim 300{ }^{\circ} \mathrm{C}[56]$.
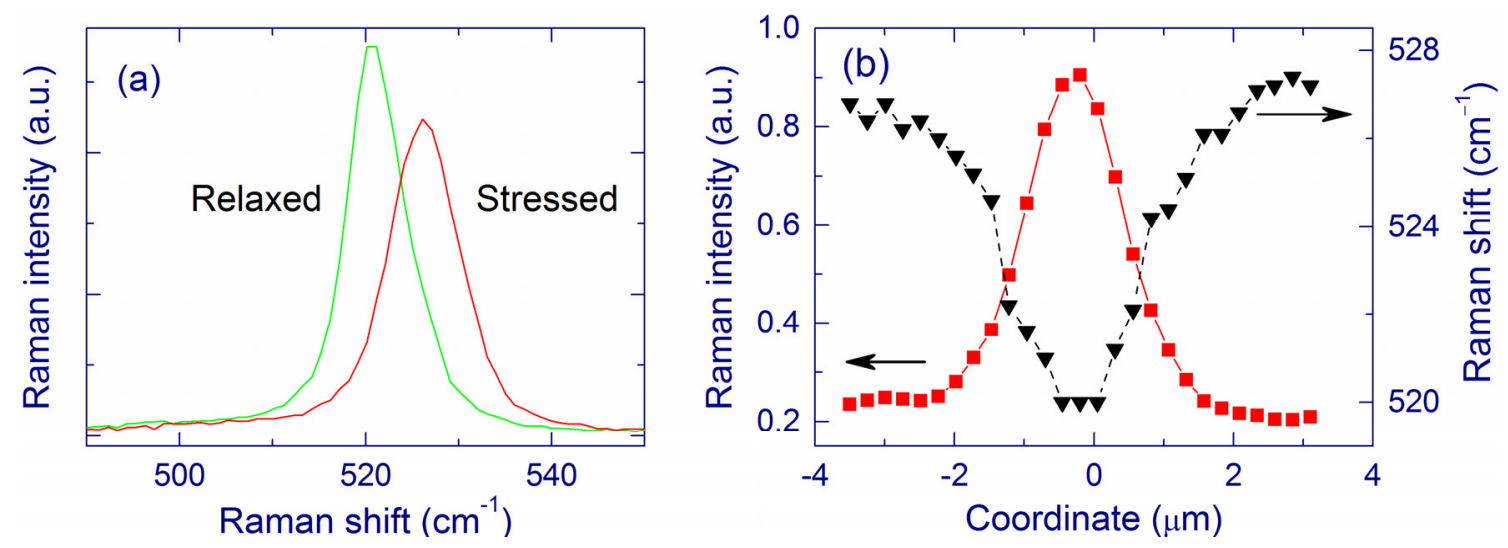

Figure 19. (a) Raman spectra of Si-nc with stress of $\sim 3 \mathrm{GPa}$ and after laser-induced relaxation (for $2-\mu \mathrm{m}$-thick $\mathrm{Si} / \mathrm{SiO}_{2} \mathrm{SL}$ ). (b) Cross-sections of the Raman band position and Raman intensity integrated in the $510-520 \mathrm{~cm}^{-1}$ region. Reproduced with permission from [151]. Copyright 2009, AIP Publishing LLC.

\section{Experimental Section}

\subsection{Samples}

Most of the samples used in this study have been prepared by MBD in the Aalto University (Finland) by S. Novikov. The $\mathrm{SiO}_{x}$ films are deposited on silica wafers. Some of the samples have a gradient of the Si content in the lateral direction, and they are called optical wedges. Due to the deposition conditions, these films also have a varying thickness $(1.5-2.5 \mu \mathrm{m})$. The advantage of the optical wedges is that the areas with different Si contents are annealed under the same conditions and the $\mathrm{O} / \mathrm{Si}$ concentration ratio $x$ can be continuously changed by selecting the film area. The wedged samples are cut in slices (5-7 cm long and $\sim 1 \mathrm{~cm}$ wide) along the $\mathrm{Si}$ content gradient and annealed in a furnace at temperatures up to $1200{ }^{\circ} \mathrm{C}$ for $1 \mathrm{~h}$ in nitrogen atmosphere. In [116], three samples covering $x$ from $\sim 1.3$ to $\sim 1.98$ are studied.

The free-standing films are prepared from the $\mathrm{SiO}_{x}$ films and $\mathrm{Si} / \mathrm{SiO}_{2} \mathrm{SL}$ sdeposited on $\mathrm{Si}$ wafers and annealed in a furnace at $1100{ }^{\circ} \mathrm{C}$ in nitrogen atmosphere. The free-standing films are made by chemical etching of the Si substrate [125,140-142]. The $\mathrm{Si} / \mathrm{SiO}_{2} \mathrm{SLs}$ are constituted of 125 or 500 
pairs of 2-nm-thick $\mathrm{Si}$ and $\mathrm{SiO}_{2}$ layers, yielding the total film thicknesses 0.5 or $2 \mu \mathrm{m}$, respectively. The thicknesses of the free-standing $\mathrm{SiO}_{x}$ films $(x \sim 1.7-1.8)$ are 0.5 and $2 \mu \mathrm{m}$.

One sample is made by Si-ion implantation into a silica substrate in the Accelerator Laboratory (University of Helsinki) [118]. The implantation of Si ions into a silica plate was done with different energies $(30,56,98,160,250$, and $400 \mathrm{keV})$ and doses to achieve a nearly constant Si excess in the $\mathrm{SiO}_{x}$ layer with a thickness of $\sim 750 \mathrm{~nm}$. The $\mathrm{O} / \mathrm{Si}$ concentration ratio $x$ estimated by XPS is $\sim 1.8$. The implanted sample is annealed at various temperatures up to $1200^{\circ} \mathrm{C}$ for $1 \mathrm{~h}$ in a nitrogen atmosphere.

\subsection{Equipment}

The main analytical techniques used in our laboratory are Raman, PL, and UV-visible absorption methods. Two setups have been used to record the Raman and PL spectra. The first setup consists of an argon-ion laser (488 nm, Melles Griot 543-AP-A01), a spectrometer (Acton SpectraPro 500I), and a charge-coupled device camera (Andor InstaSpec IV). The laser beam is focused with a lens to a spot with a diameter of $\sim 40 \mu \mathrm{m}$. The second setup allows us to perform micro-Raman and micro-PL measurements. It consists of a confocal Raman microscope (LabRAM HR800, Horiba Jobin Yvon) equipped with an argon-ion laser $(488 \mathrm{~nm})$. The size of the laser spot in the microscopic measurements is down to $\sim 1 \mu \mathrm{m}$. For both setups, the spectral resolution is $\sim 2 \mathrm{~cm}^{-1}$ and $10 \mathrm{~cm}^{-1}$ for Raman and PL measurements, respectively. The Raman bands of the films annealed at $\geq 1000{ }^{\circ} \mathrm{C}$ are fitted with two or three Gaussians. The position of the Raman band of Si-nc is obtained from the position of the higher-frequency Gaussian $\left(\sim 518-530 \mathrm{~cm}^{-1}\right)$. The PL spectra are corrected for the spectral sensitivity of the apparatus. The Raman and PL intensities are normalized by the effective thickness $\int_{0}^{d} e^{(-2 a x)} d x$ and $\int_{0}^{d} e^{(-a x)} d x$, respectively, where the same absorption coefficients $\alpha$ is assumed for the laser and Raman light, the absorption for the PL is neglected, and $d$ is the film thickness [55].

Laser annealing of free-standing films is performed using a TEM 00 beam of an argon-ion laser (488 nm, power up to $110 \mathrm{~mW}$ at the sample) focused to spots with diameters ranging from $\sim 1$ to $\sim 40 \mu \mathrm{m}$. The exposure time is mainly $\sim 1 \mathrm{~s}$, but 10 -ms and 100 -s exposures is also used in some experiments. Most of laser-annealing studies are performed in air; however, a number of measurements are done in $\mathrm{O}_{2}, \mathrm{~N}_{2}$, and Ar atmospheres.

The absorption coefficient is obtained from the transmission and reflection spectra [152], recorded with a fiber-optics spectrometer (SD2000, Ocean Optics) and a broadband light source (DH-2000, Top Sensor Systems) or by measuring transmitted and reflected laser light using a power meter (NOVA II, OPHIR). The micro-transmission measurements of the 488-nm light are carried out in the LabRAM microscope with a large-area photodiode located behind the sample.

Additional measurements by other methods have been available from cooperation: XPS [115-118] including measurements with high spatial resolution [140], FTIR spectroscopy with high spatial resolution [140], TEM including bright-field scanning (BF), STEM and EFTEM [140,141], EELS [140], SEM and EDS [141,142], AFM [141,142], and $m$-line measurements [115]. The equipment used in these methods is described in the given references. 


\subsection{PL-Filtering Effect}

The method to measure the optical properties of the films utilizes the PL-filtering effect. The PL filtering is observed when the PL spectra are measured in a direction along the film surface. In this case, narrow and polarized spectral peaks are detected (Figure 20a). The PL filtering has been observed in various silica films containing Si-nc [49,57,59,79,85-87,94].

The PL-filtering effect can be interpreted in terms of delocalization of guided modes near the cut-off frequency $[49,57,79,85]$. The minimal losses occur near the cut-off wavelengths, for which the delocalized light travels mostly in the transparent substrate rather than in the absorbing film. Thus, the narrow peaks seen in the PL spectra obtained in the waveguiding direction correspond to the cut-off wavelengths. The mode localization is a function of the generalized frequency parameter written for an asymmetrical planar waveguide in the form:

$$
V=2 \pi \sqrt{\left(n_{1}^{2}-n_{2}^{2}\right)} d / \lambda
$$

where $n_{1}$ and $n_{2}$ are the refractive indexes of the film and substrate, respectively, $d$ is the film thickness, and $\lambda$ is the wavelength. This equation is obtained assuming weak-guiding approximation $\left(n_{1}-n_{2}\right) \ll 1$ [127]. For the cut-off condition $V=(2 m+1) \pi / 2$, Equation (1) yields the cut-off wavelengths.

Figure $20 \mathrm{~b}$ shows a typical transmission spectrum of an annealed $\mathrm{SiO}_{1.5}$ film on a silica substrate. The spacing of the interference patterns $\left(\right.$ in $^{-1}$ ) can be written as:

$$
\delta v=1 / 2 n_{1} d
$$

which provides the optical thickness $(n d)$. The numerical information can be extracted from the transmission spectrum by fitting it, for example, with the following empirical function:

$$
T(\lambda)=P_{7}+P_{1}\left(1-\frac{P_{3}}{\lambda-P_{2}}\right)\left[1-P_{4} \sin \left(\frac{P_{5}}{\lambda}+P_{6}\right)\right]
$$

where $T$ is the transmittance, and $P_{i}$ are the fitting parameters [49]. In particular, the $P_{5}$ parameter is connected with the period of the oscillations, i.e., with the optical thickness. The TE refractive index is relevant to transmission spectra. By using Equation (1) for the spacing between the TE peaks and Equation (2), the TE refractive index and the film thickness can be extracted [85]. Next, the obtained layer thickness together with the spacing between the TM peak positions is used to calculate the TM refractive index. The obtained thickness is also used to calculate the absorption (extinction) coefficient from the transmission and reflection measurements.

The model described above is called a simple model because it uses a few simplifications: (i) Equation (1) corresponds to the weak-guiding approximation $\left(n_{1}-n_{2}\right) \ll 1$; (ii) the procedure uses only the interval between the cut-off peaks disregarding their actual positions; (iii) the material dispersion is neglected; and (iv) a step-like profile is assumed for the refractive index. 

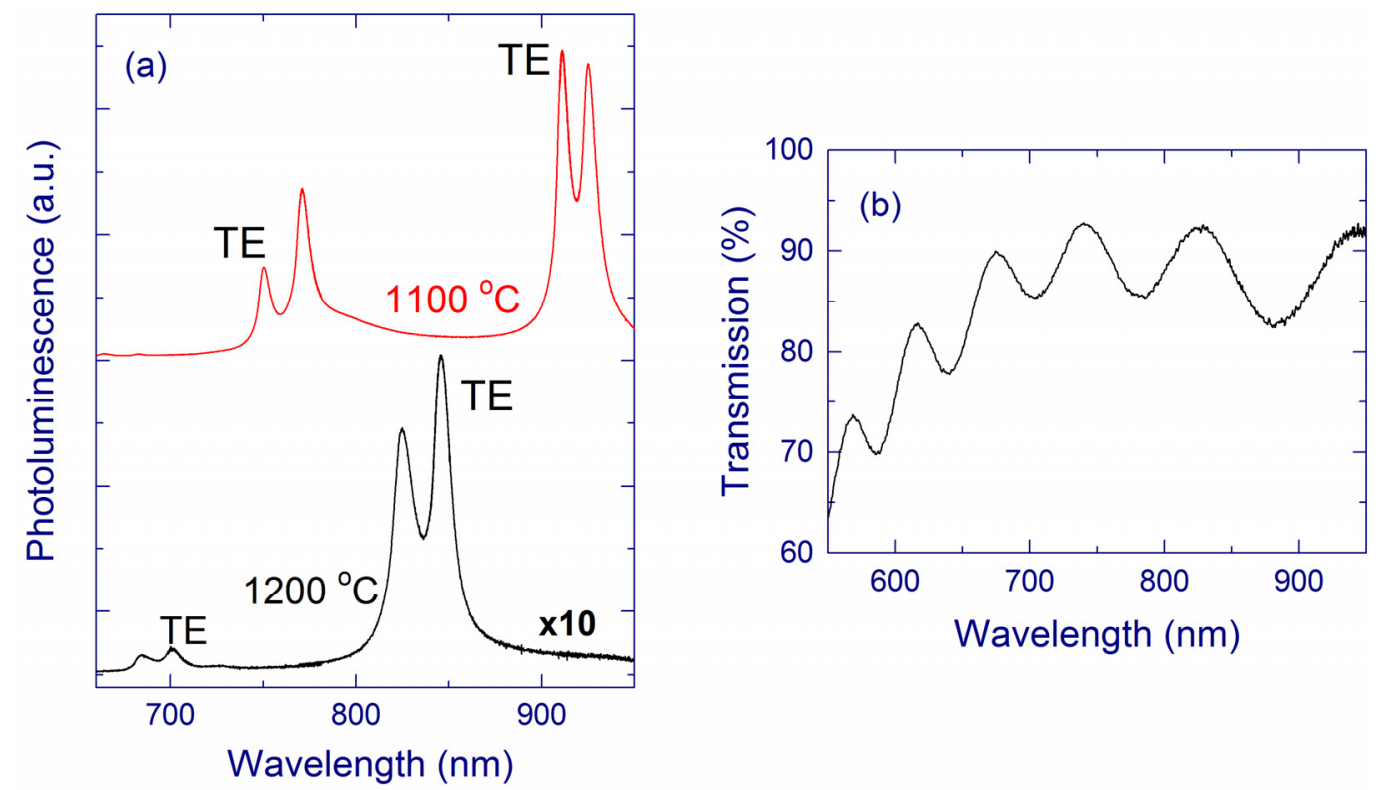

Figure 20. (a) Cut-off spectra of a $\mathrm{SiO}_{1.5}$ film annealed at 1100 and $1200{ }^{\circ} \mathrm{C}$, measured from the sample edge along the film. The excitation spot was at a distance of several millimeters from the sample edge. The positions of the TE peaks are marked and the other bands have the TM polarization. The TE and TM peaks have polarization parallel and perpendicular to the film surface, respectively. (b) Transmission spectrum of the $\mathrm{SiO}_{1.5}$ film annealed at $1100{ }^{\circ} \mathrm{C}$. Reproduced with permission from [115]. Copyright 2008, AIP Publishing LLC.

To take the dispersion of the material into account, the transmission spectra can be fitted with a function similar to Equation (3) but containing an additional term describing the wavelength dependence of the spacing $\delta v$ [115]. Equations (1) and (2) are then solved taking the dispersion correction into account. The results show that the dispersion corrections for the refractive index and the film thickness are within experimental errors (Figure 21).

The refractive index and the film thickness can be also calculated using the model of reflection from a multilayer system [153], which does not limit the refractive index (exact equations). The analysis includes both spacing and positions of the cut-off peaks. Equation (3) with an additional term describing the material dispersion is also used in this case [115]. Again, the differences from the simple model are within the experimental errors ("exact" in Figure 21). Using the XPS data for a $\mathrm{SiO}_{1.55}$ film annealed at $1100{ }^{\circ} \mathrm{C}$, it has been concluded that the use of smooth index profile of the refractive index is not crucial to obtain good description of the delocalized cut-off modes. These results suggest that the simple model is sufficient to achieve adequate estimates of the refractive index and film thickness. The $m$-line measurements lead to the results very similar to those obtained by the PL-filtering effect.

PL-filtering effect has also been interpreted in terms of substrate leaky (or radiation) modes of a planar waveguide [86,87]. In this model, the regular guided modes experience greater loss propagating over a macroscopic distance from the place of creation towards the sample edge. The most likely source of the loss is absorption and scattering by nanocrystals, as well as scattering at the "interface" between the core and cladding layers. In contrast, the beams responsible for generating the substrate 
modes travel shorter distances through the core region, and the substrate mode undergoes virtually no loss in traveling to the substrate facet. There seems to be no contradiction between the descriptions based on mode delocalization and substrate leaky modes. In particular, both of these models explain the PL-filtering effect based on a smaller absorption in the substrate compared to the film containing Si-nc.

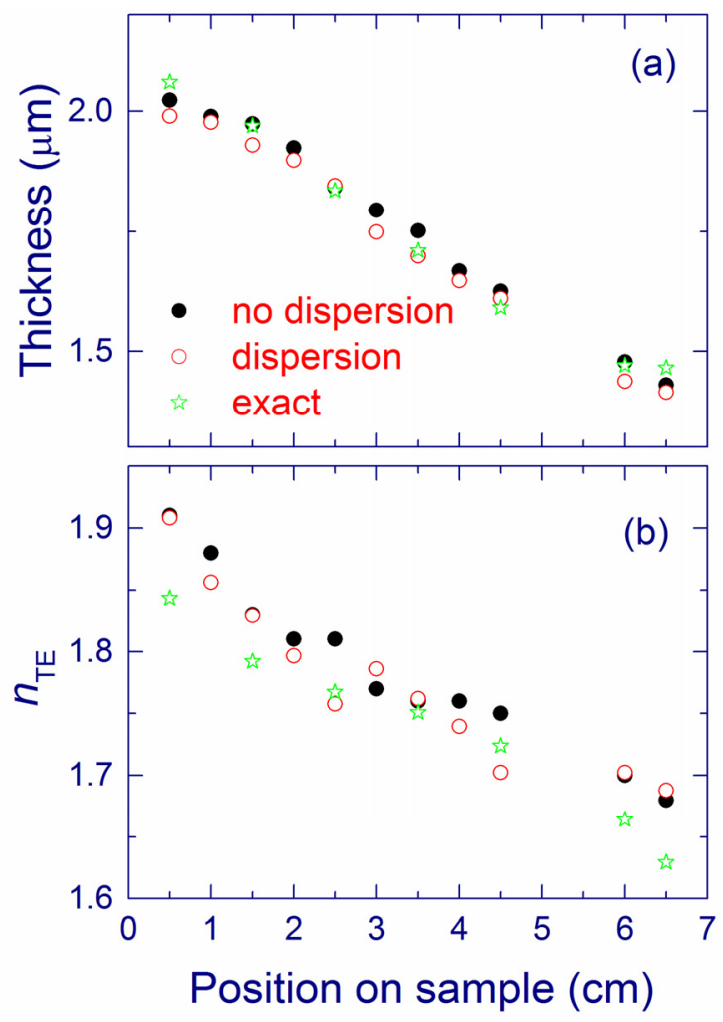

Figure 21. Optical parameters of a $\mathrm{SiO}_{x}$ optical wedge $(1.5<x<1.8)$ obtained with different models: simple model (solid circles), taking dispersion into account (open circles), and using the exact equations [153] (stars). Reproduced with permission from [115]. Copyright 2008, AIP Publishing LLC.

\subsection{X-Ray Photoelectron Spectroscopy}

XPS measurements provide detailed information about the elemental and chemical composition of the material. Since only a thin layer of the sample $(\leq 10 \mathrm{~nm})$ is investigated with this method, the sample is sputtered with $\mathrm{Ar}^{+}$ions to obtain information about the bulk material.

In the case of $\mathrm{SiO}_{x}$ films, the $\mathrm{Si} 2 p$ spectrum is fitted by several peaks to obtain the amounts of $\mathrm{Si}$ in different oxidation states. Three peaks are often used to describe $\mathrm{Si}$ atoms bonded as in crystalline $\mathrm{Si}$ (elemental $\mathrm{Si}$ ), "SiO" (mixture of various suboxides), and $\mathrm{SiO}_{2}$ materials (Figure 22). More complicated fitting with five peaks can be used to obtain the five oxidation states of Si (elemental Si, $\mathrm{Si}_{2} \mathrm{O}, \mathrm{SiO}, \mathrm{Si}_{2} \mathrm{O}_{3}$, and $\mathrm{SiO}_{2}$ ) [154-156]. For our samples, the fittings with three and five peaks give similar amounts of elemental $\mathrm{Si}$ and $\mathrm{SiO}_{2}$ [116]. Moreover, the use of five fitting curves is problematic for low Si content. 


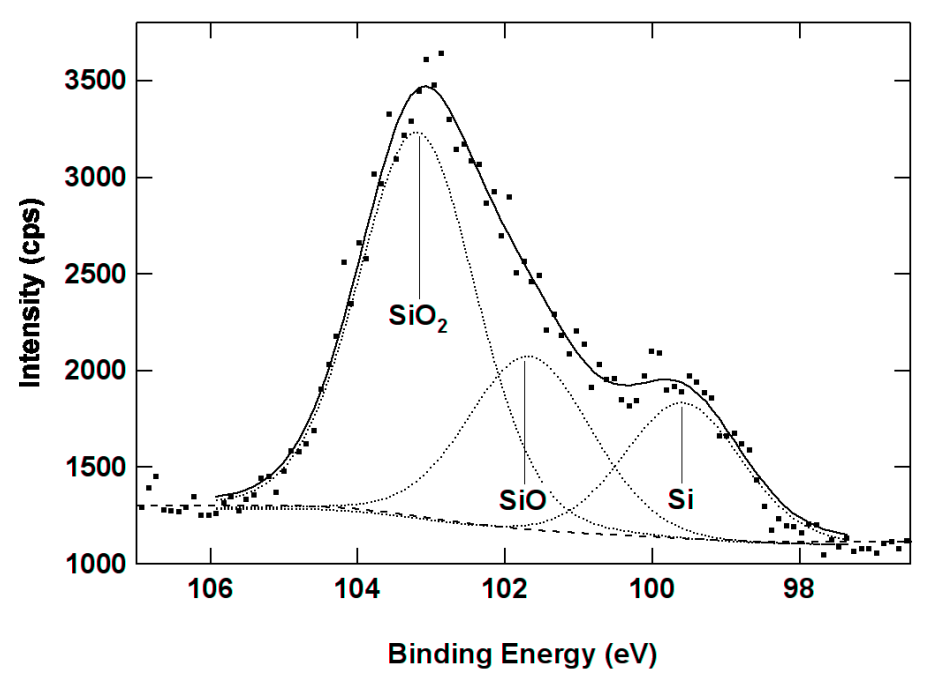

Figure 22. Typical XPS spectrum of a $\mathrm{SiO}_{x}$ film fitted with three Gaussians corresponding to $\mathrm{Si}$ bonded as in elemental $\mathrm{Si}, \mathrm{SiO}$ (mixture of suboxides), and $\mathrm{SiO}_{2}$. Figure courtesy of Jouko Lahtinen. Reproduced with permission from [115]. Copyright 2008, AIP Publishing LLC.

\subsection{Effective Medium Approximation}

The effective medium approximation (Bruggeman theory) connects the effective dielectric constant with the dielectric constants of the three structural components and their volumetric fractions [157]:

$$
a\left(\frac{\varepsilon_{a}-\varepsilon_{m}}{\varepsilon_{a}+2 \varepsilon_{m}}\right)+b\left(\frac{\varepsilon_{b}-\varepsilon_{m}}{\varepsilon_{b}+2 \varepsilon_{m}}\right)+c\left(\frac{\varepsilon_{c}-\varepsilon_{m}}{\varepsilon_{c}+2 \varepsilon_{m}}\right)=0
$$

where $a, b$, and $c$ are the volumetric fractions of each component, $\varepsilon a, b, c$ are the corresponding dielectric constants, and $\varepsilon_{m}$ is the effective dielectric constant. The effective medium approximation is employed to calculate the complex refractive index $n^{*}=n+i k$ (where $k$ is the extinction coefficient) using the chemical compositions ( $\mathrm{Si}, \mathrm{SiO}$, and $\mathrm{SiO}_{2}$ ) obtained from the XPS measurements.

\subsection{Temperature Measurements}

Laser-induced temperature $(T)$ can be measured in-situ using the intensities of the Stokes $\left(I_{\mathrm{S}}\right)$ and anti-Stokes $\left(I_{\mathrm{AS}}\right)$ Raman bands (Figure 23$)$ and the relation:

$$
\frac{I_{\mathrm{AS}}}{I_{\mathrm{S}}}=A e^{\left(-E_{\mathrm{R}} / k T\right)}
$$

where $E_{\mathrm{R}}$ is the phonon energy and $A$ is a correction coefficient $[95,139]$. The coefficient $A \sim 0.95$ is obtained by measuring the laser-induced temperature of a Si wafer as a function of the laser power and assuming room temperature in the irradiated volume at zero laser power. 


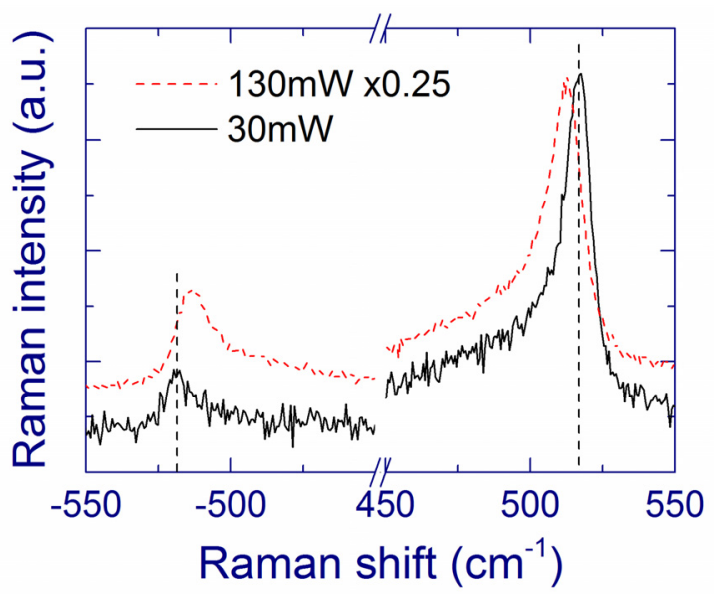

Figure 23. Raman spectra of a $\mathrm{SiO}_{1.3}$ film on a silica substrate measured with two laser powers $(\sim 30$ and $\sim 130 \mathrm{~mW}$ focused to a $\sim 40 \mu \mathrm{m}$ spot). The spectra are vertically shifted and scaled for better presentation.

\subsection{Optical Absorption}

The film absorption is obtained by measuring transmission and reflection in ultraviolet and visible regions using a broadband light source. The band gap of semiconductors can be obtained from the absorption spectra using the Tauc relation, according to which, the absorption spectrum obeys the following equation $[88,158]$ :

$$
\sqrt{\alpha \cdot h v}=A\left(h v-E_{\text {Tauc }}\right)
$$

where $A$ is a correction coefficient. Function $\sqrt{\alpha \cdot h v}$ is plotted versus $h v$ and fitted with a straight line. The value of the optical gap $\left(E_{\text {Tauc }}\right)$ is obtained from the intercept of the linear fit with the abscissa.

\section{Conclusions}

Optical and structural properties of Si-nc in silica films have been described. In order to prepare Si-nc, $\operatorname{SiO}_{x}(x<2)$ films are annealed in a furnace at temperatures up to $1200{ }^{\circ} \mathrm{C}$. For different $\mathrm{Si}$ contents, the Si-nc Raman signal and the absorption coefficient are proportional to the amount of elemental Si detected by XPS. On the other hand, the Raman scattering cross-section of elemental Si is about three times smaller than that of crystalline Si. This difference can be explained by the presence of small Si-nc ( $<2 \mathrm{~nm})$ and/or by the different properties of bulk and nanoscale Si.

The measured optical properties of $\mathrm{SiO}_{x}$ films are compared with the values estimated by the effective medium approximation using the XPS results. A good agreement is found between the measured and calculated refractive index. The results for absorption suggest high transparency of nanoscale suboxide in the annealed samples. Thermal annealing increases the degree of $\mathrm{Si}$ crystallization; however, the crystallization is not complete after annealing at $1200{ }^{\circ} \mathrm{C}$. The extinction coefficient of elemental $\mathrm{Si}$ is found to be between the values of crystalline and amorphous $\mathrm{Si}$.

The PL quantum yield increases as the amount of elemental Si decreases. It follows that the 1.5-eV PL from the $\mathrm{SiO}_{x}$ films annealed above $1000{ }^{\circ} \mathrm{C}$ is probably not directly from Si-nc responsible for absorption and detected by Raman spectroscopy. According to these results, the light-emitting centers 
may be small $(\sim 1 \mathrm{~nm})$ oxidized Si grains or oxygen-related defects, which are not detectable by Raman spectroscopy. This conclusion is in agreement with the theoretical simulations [114].

The as-prepared $\mathrm{SiO}_{x}$ films deposited by MBD and ion implantation are structurally and optically very different. Relatively large amorphous clusters $(>2 \mathrm{~nm})$ are present in the MBD films whereas they are absent in the implanted samples as suggested by absorption and Raman spectroscopy. After annealing at $\geq 1100{ }^{\circ} \mathrm{C}$, these two kinds of samples become similar and possess comparable amounts of Si-nc.

Laser-induced thermal effects are found for $\mathrm{SiO}_{x}$ films on silica substrates when illuminated by focused laser light. In our experiments, a temperature of $\sim 350{ }^{\circ} \mathrm{C}$ was estimated for $\mathrm{SiO}_{x}$ films $(x \sim 1.3)$ on a silica substrate for 488-nm laser intensity of $\sim 10^{4} \mathrm{~W} \mathrm{~cm}^{-2}$, which decreases the Raman shift by $\sim 6 \mathrm{~cm}^{-1}$. This effect should be taken into account in optical measurements using focused laser beams, especially in Raman microscopy.

CW laser light can produce very high temperatures in free-standing $\mathrm{SiO}_{x}$ films and $\mathrm{Si} / \mathrm{SiO}_{2} \mathrm{SLs}$, which strongly changes their structure and optical properties. The center of a laser-annealed area is very transparent and presumably consists of amorphous $\mathrm{SiO}_{2}$. Large Si-nc (up to $300 \mathrm{~nm}$ ) are observed in the ring around the central region. These Si-nc possess high absorption of visible light and they are typically under compressive stress, which is connected with the crystallization from the liquid phase. Some of these large Si-nc exhibit surface features that are formed by eruption of pressurized Si from the film. Some of these "surface" Si-nc are removed from the film leading to holes of similar sizes. The presence of oxygen in the laser-annealing atmosphere decreases the amount of removed $\mathrm{Si}$ particles most probably due to oxidation of their surface.

The structure of laser-annealed areas is explained by thermodiffusion in temperature gradient, which means the macroscopic $\mathrm{Si}-\mathrm{SiO}_{2}$ phase separation. Comparison of the structure of central regions for laser annealing in oxygen, air, and inert atmospheres excludes the dominating effect of $\mathrm{Si}$ oxidation, thus, supporting the mechanism of the macroscopic $\mathrm{Si}-\mathrm{SiO}_{2}$ phase separation.

By using a strongly focused laser beam, the structural changes in the free-standing films can be made in areas of submicron sizes. A concept of high-density non-volatile optical memory with superior thermal stability is demonstrated where the information can be read, rewritten and erased by optical means.

\section{Acknowledgments}

The authors thank all colleagues who contributed to the results described in this article and whose names appear in the joint publications.

\section{Author Contributions}

TN and LK have written this review.

\section{Conflicts of Interest}

The authors declare no conflict of interest. 


\section{References}

1. Silicon Nanophotonics: Basic Principles, Present Status and Perspectives; Khriachtchev, L., Ed.; Pan Stanford: Singapore, Singapore, 2008.

2. Silicon Photonics II: Components and Integration; Lockwood, D.J., Pavesi, L., Eds.; Springer-Verlag: Berlin, Germany, 2011.

3. Nanostructures in Electronics and Photonics; Rahman, F., Ed.; Pan Stanford: Singapore, Singapore, 2008.

4. Yariv, A.; Yeh, P. Photonics: Optical Electronics in Modern Communications; Oxford University Press: Oxford, UK, 2007.

5. Jalali, B.; Fathpour, S. Silicon photonics. J. Lightwave Technol. 2006, 24, 4600-4615.

6. Soref, R. The past, present, and future of silicon photonics. IEEE J. Sel. Top. Quant. Electron. 2006, 12, 1678-1687.

7. Daldosso, N.; Pavesi, L. Nanosilicon photonics. Laser Photonics Rev. 2009, 3, 508-534.

8. Silicon Photonics: The State of the Art; Reed, G.T., Ed.; John Wiley \& Sons, Ltd: Chichester, UK, 2008.

9. Pavesi, L. Will silicon be the photonic material of the third millennium? J. Phys. Cond. Matter 2003, 15, R1169-R1196.

10. Lockwood, D.J. Light emission in silicon nanostructures. NATO Asi Ser. Ser. E 1998, 348, 185-209.

11. Lockwood, D.J.; Pavesi, L. Silicon fundamentals for photonics applications. Top. Appl. Phys. 2004, 94, 1-50.

12. Delley, B.; Steigmeier, E.F. Quantum confinement in Si nanocrystals. Phys. Rev. B 1993, 47, 1397-1400.

13. Ogut, S.; Chelikowsky, J.R.; Louie, S.G. Quantum confinement and optical gaps in Si nanocrystals. Phys. Rev. Lett. 1997, 79, 1770-1773.

14. Balberg, I. Electrical transport mechanisms in three dimensional ensembles of silicon quantum dots. J. App. Phys. 2011, 110, 061301.

15. Canham, L.T. Silicon quantum wire array fabrication by electrochemical and chemical dissolution of wafers. Appl. Phys. Lett. 1990, 57, 1046-1048.

16. Cullis, A.G.; Canham, L.T. Visible-light emission due to quantum size effects in highly porous crystalline silicon. Nature 1991, 353, 335-338.

17. Gardelis, S.; Rimmer, J.S.; Dawson, P.; Hamilton, B.; Kubiak, R.A.; Whall, T.E.; Parker, E.H.C. Evidence for quantum confinement in the photoluminescence of porous Si and SiGe. Appl. Phys. Lett. 1991, 59, 2118-2120.

18. Bsiesy, A.; Vial, J.C.; Gaspard, F.; Herino, R.; Ligeon, M.; Muller, F.; Romestain, R.; Wasiela, A.; Halimaoui, A.; Bomchil, G. Photoluminescence of high porosity and of electrochemically oxidized porous silicon layers. Surf. Sci. 1991, 254, 195-200.

19. Delerue, C.; Allan, G.; Lannoo, M. Theoretical aspects of the luminescence of porous silicon. Phys. Rev. B 1993, 48, 11024-11036.

20. Prokes, S.M. Light emission in thermally oxidized porous silicon: Evidence for oxide-related luminescence. Appl. Phys. Lett. 1993, 62, 3244-3246. 
21. Gole, J.L.; Dudel, F.P.; Grantier, D.; Dixon, D.A. Origin of porous silicon photoluminescence: Evidence for a surface bound oxyhydride-like emitter. Phys. Rev. B 1997, 56, 2137-2153.

22. Wolkin, M.V.; Jorne, J.; Fauchet, P.M.; Allan, G.; Delerue, C. Electronic states and luminescence in porous silicon quantum dots: The role of oxygen. Phys. Rev. Lett. 1999, 82, 197-200.

23. Ledoux, G.; Gong, J.; Huisken, F.; Guillois, O.; Reynaud, C. Photoluminescence of size-separated silicon nanocrystals: Confirmation of quantum confinement. Appl. Phys. Lett. 2002, 80, 4834-4836.

24. Hannah, D.C.; Yang, J.H.; Podsiadlo, P.; Chan, M.K.Y.; Demortiere, A.; Gosztola, D.J.; Prakapenka, V.B.; Schatz, G.C.; Kortshagen, U.; Schaller, R.D. On the origin of photoluminescence in silicon nanocrystals: Pressure-dependent structural and optical studies. Nano Lett. 2012, 12, 4200-4205.

25. Kim, T.Y.; Park, N.M.; Kim, K.H.; Sung, G.Y.; Ok, Y.W.; Seong, T.Y.; Choi, C.J. Quantum confinement effect of silicon nanocrystals in situ grown in silicon nitride films. Appl. Phys. Lett. 2004, 85, 5355-5357.

26. Rezgui, B.; Sibai, A.; Nychyporuk, T.; Lemiti, M.; Bremond, G. Photoluminescence and optical absorption properties of silicon quantum dots embedded in Si-rich silicon nitride matrices. J. Lumin. 2009, 129, 1744-1746.

27. Khriachtchev, L.; Novikov, S.; Lahtinen, J. Thermal annealing of $\mathrm{Si} / \mathrm{SiO}_{2}$ materials: Modification of structural and photoluminescence emission properties. J. Appl. Phys. 2002, 92, 5856-5862.

28. Lu, Z.H.; Lockwood, D.J.; Baribeau, J.M. Quantum confinement and light emission in $\mathrm{SiO}_{2} / \mathrm{Si}$ superlattices. Nature 1995, 378, 258-260.

29. Zhuravlev, K.S.; Gilinsky, A.M.; Kobitsky, A.Y. Mechanism of photoluminescence of Si nanocrystals fabricated in $\mathrm{SiO}_{2}$ matrix. Appl. Phys. Lett. 1998, 73, 2962-2964.

30. Dhara, S.; Lu, C.Y.; Nair, K.G.M.; Chen, K.H.; Chen, C.P.; Huang, Y.F.; David, C.; Chen, L.C.; Raj, B. Mechanism of bright red emission in Si nanoclusters. Nanotechnology 2008, 19, 395401.

31. Garrido Fernandez, B.; Lopez, M.; Garcia, C.; Perez-Rodriguez, A.; Morante, J.R.; Bonafos, C.; Carrada, M.; Claverie, A. Influence of average size and interface passivation on the spectral emission of Si nanocrystals embedded in $\mathrm{SiO}_{2}$. J. Appl. Phys. 2002, 91, 798-807.

32. Guha, S. Characterization of $\mathrm{Si}^{+}$ion-implanted $\mathrm{SiO}_{2}$ films and silica glasses. J. Appl. Phys. 1998, $84,5210-5217$.

33. Mirabella, S.; Agosta, R.; Franzo, G.; Crupi, I.; Miritello, M.; Lo Savio, R.; Di Stefano, M.A.; Di Marco, S.; Simone, F.; Terrasi, A. Light absorption in silicon quantum dots embedded in silica. J. Appl. Phys. 2009, 106, 103505.

34. Franzo, G.; Miritello, M.; Boninelli, S.; Lo Savio, R.; Grimaldi, M.G.; Priolo, F.; Iacona, F.; Nicotra, G.; Spinella, C.; Coffa, S. Microstructural evolution of $\mathrm{SiO}_{x}$ films and its effect on the luminescence of Si nanoclusters. J. Appl. Phys. 2008, 104, 094306.

35. Ternon, C.; Dufour, C.; Gourbilleau, F.; Rizk, R. Roles of interfaces in nanostructured silicon luminescence. Eur. Phys. J. B 2004, 41, 325-332.

36. Alonso, M.I.; Marcus, I.C.; Garriga, M.; Goñi, A.R.; Jedrzejewski, J.; Balberg, I. Evidence of quantum confinement effects on interband optical transitions in Si nanocrystals. Phys. Rev. B 2010, $82,045302$. 
37. Roussel, M.; Talbot, E.; Pareige, P.; Gourbilleau, F. Influence of the supersaturation on Si diffusion and growth of Si nanoparticles in silicon-rich silica. J. Appl. Phys. 2013, 113, 063519.

38. Antonova, I.V.; Gulyaev, M.; Savir, E.; Jedrzejewski, J.; Balberg, I. Charge storage, photoluminescence, and cluster statistics in ensembles of Si quantum dots. Phys. Rev. B 2008, 77, 125318.

39. Balberg, I.; Savir, E.; Jedrzejewski, J.; Nassiopoulou, A.G.; Gardelis, S. Fundamental transport processes in ensembles of silicon quantum dots. Phys. Rev. B 2007, 75, 235329.

40. Iacona, F.; Franzo, G.; Spinella, C. Correlation between luminescence and structural properties of Si nanocrystals. J. Appl. Phys. 2000, 87, 1295-1303.

41. Trwoga, P.F.; Kenyon, A.J.; Pitt, C.W. Modeling the contribution of quantum confinement to luminescence from silicon nanoclusters. J. Appl. Phys. 1998, 83, 3789-3794.

42. Boninelli, S.; Iacona, F.; Franzo, G.; Bongiorno, C.; Spinella, C.; Priolo, F. Formation, evolution and photoluminescence properties of Si nanoclusters. J. Phys.: Condens. Matter 2007, 19, 225003.

43. Nassiopoulou, A.G. Encyclopedia of Nanoscience and Nanotechnology; Nalwa, H.S., Ed.; American Scientific: Valencia, Spain, 2004; Volume 9, pp. 793-813.

44. Gardelis, S.; Nassiopoulou, A.G.; Manousiadis, P.; Milita, S.; Gkanatsiou, A.; Frangis, N.; Lioutas, C.B. Structural and optical characterization of two-dimensional arrays of Si nanocrystals embedded in $\mathrm{SiO}_{2}$ for photovoltaic applications. J. Appl. Phys. 2012, 111, 083536.

45. Gardelis, S.; Nassiopoulou, A.G.; Vouroutzis, N.; Frangis, N. Effect of exciton migration on the light emission properties in silicon nanocrystal ensembles. J. Appl. Phys. 2009, 105, 113509.

46. Hiller, D.; Goetze, S.; Munnik, F.; Jivanescu, M.; Gerlach, J.W.; Vogt, J.; Pippel, E.; Zakharov, N.; Stesmans, A.; Zacharias, M. Nitrogen at the Si-nanocrystal $/ \mathrm{SiO}_{2}$ interface and its influence on luminescence and interface defects. Phys. Rev. B 2010, 82, 195401.

47. Seguini, G.; Castro, C.; Schamm-Chardon, S.; BenAssayag, G.; Pellegrino, P.; Perego, M. Scaling size of the interplay between quantum confinement and surface related effects in nanostructured silicon. Appl. Phys. Lett. 2013, 103, 023103.

48. Khriachtchev, L.; Räsänen, M.; Novikov, S.; Pavesi, L. Systematic correlation between Raman spectra, photoluminescence intensity, and absorption coefficient of silica layers containing $\mathrm{Si}$ nanocrystals. Appl. Phys. Lett. 2004, 85, 1511-1513.

49. Khriachtchev, L.; Räsänen, M.; Novikov, S.; Lahtinen, J. Tunable wavelength-selective waveguiding of photoluminescence in Si-rich silica optical wedges. J. Appl. Phys. 2004, 95, 7592-7601.

50. Zacharias, M.; Blasing, J.; Veit, P.; Tsybeskov, L.; Hirschman, K.; Fauchet, P.M. Thermal crystallization of amorphous $\mathrm{Si} / \mathrm{SiO}_{2}$ superlattices. Appl. Phys. Lett. 1999, 74, 2614-2616.

51. Zacharias, M.; Heitmann, J.; Scholz, R.; Kahler, U.; Schmidt, M.; Blasing, J. Size-controlled highly luminescent silicon nanocrystals: A $\mathrm{SiO} / \mathrm{SiO}_{2}$ superlattice approach. Appl. Phys. Lett. 2002, 80, 661-663.

52. Godefroo, S.; Hayne, M.; Jivanescu, M.; Stesmans, A.; Zacharias, M.; Lebedev, O.I.; van Tendeloo, G.; Moshchalkov, V.V. Classification and control of the origin of photoluminescence from Si nanocrystals. Nat. Nanotechnol. 2008, 3, 174-178.

53. Hiller, D.; Jivanescu, M.; Stesmans, A.; Zacharias, M. P-b(0) centers at the Si-nanocrystal/ $/ \mathrm{SiO}_{2}$ interface as the dominant photoluminescence quenching defect. J. Appl. Phys. 2010, 107, 084309. 
54. Novikov, S.V.; Sinkkonen, J.; Kilpela, O.; Gastev, S.V. Visible light emission from MBD-grown $\mathrm{Si} / \mathrm{SiO}_{2}$ superlattices. J. Cryst. Growth 1997, 175, 514-518.

55. Khriachtchev, L.; Räsänen, M.; Novikov, S.; Kilpela, O.; Sinkkonen, J. Raman scattering from very thin $\mathrm{Si}$ layers of $\mathrm{Si} / \mathrm{SiO}_{2}$ superlattices: Experimental evidence of structural modification in the 0.8-3.5 nm thickness region. J. Appl. Phys. 1999, 86, 5601-5608.

56. Khriachtchev, L.; Räsänen, M.; Novikov, S. Laser-controlled stress of Si nanocrystals in a free-standing $\mathrm{Si} / \mathrm{SiO}_{2}$ superlattice. Appl. Phys. Lett. 2006, 88, 013102.

57. Khriachtchev, L.; Novikov, S.; Lahtinen, J.; Räsänen, M. Wavelength-selective optical waveguiding of photoluminescence in a thermally annealed $\mathrm{Si} / \mathrm{SiO}_{2}$ superlattice. J. Phys.: Condens. Matter 2004, 16, 3219-3228.

58. Pavesi, L.; Dal Negro, L.; Mazzoleni, C.; Franzo, G.; Priolo, F. Optical gain in silicon nanocrystals. Nature 2000, 408, 440-444.

59. Khriachtchev, L.; Räsänen, M.; Novikov, S.; Sinkkonen, J. Optical gain in $\mathrm{Si} / \mathrm{SiO}_{2}$ lattice: Experimental evidence with nanosecond pulses. Appl. Phys. Lett. 2001, 79, 1249-1251.

60. Koshida, N.; Koyama, H. Visible electroluminescence from porous silicon. Appl. Phys. Lett. 1992, 60, 347-349.

61. Franzo, G.; Irrera, A.; Moreira, E.C.; Miritello, M.; Iacona, F.; Sanfilippo, D.; Di Stefano, G.; Fallica, P.G.; Priolo, F. Electroluminescence of silicon nanocrystals in MOS structures. Appl. Phys. A 2002, 74, 1-5.

62. Zhou, F.L.; Head, J.D. Role of $\mathrm{Si}=\mathrm{O}$ in the photoluminescence of porous silicon. J. Phys. Chem. B 2000, 104, 9981-9986.

63. Takeoka, S.; Fujii, M.; Hayashi, S. Size-dependent photoluminescence from surface-oxidized Si nanocrystals in a weak confinement regime. Phys. Rev. B 2000, 62, 16820-16825.

64. Nesheva, D.; Raptis, C.; Perakis, A.; Bineva, I.; Aneva, Z.; Levi, Z.; Alexandrova, S.; Hofmeister, H. Raman scattering and photoluminescence from Si nanoparticles in annealed $\mathrm{SiO}_{x}$ thin films. J. Appl. Phys. 2002, 92, 4678-4683.

65. Lioudakis, E.; Othonos, A.; Hadjisavvas, G.C.; Kelires, P.C.; Nassiopoulou, A.G. Quantum confinement and interface structure of $\mathrm{Si}$ nanocrystals of sizes $3-5 \mathrm{~nm}$ embedded in a-SiO 2 . Phys. E 2007, 38, 128-134.

66. Garcia, C.; Garrido, B.; Pellegrino, P.; Ferre, R.; Moreno, J.A.; Morante, J.R.; Pavesi, L.; Cazzanelli, M. Size dependence of lifetime and absorption cross section of Si nanocrystals embedded in $\mathrm{SiO}_{2}$. Appl. Phys. Lett. 2003, 82, 1595-1597.

67. Kanemitsu, Y.; Ogawa, T.; Shiraishi, K.; Takeda, K. Visible photoluminescence from oxidized Si nanometer-sized spheres: Exciton confinement on a spherical shell. Phys. Rev. B 1993, 48, 4883-4886.

68. Kanemitsu, Y.; Uto, H.; Masumoto, Y.; Matsumoto, T.; Futagi, T.; Mimura, H. Microstructure and optical properties of free-standing porous silicon films: Size dependence of absorption spectra in Si nanometer-sized srystallites. Phys. Rev. B 1993, 48, 2827-2830.

69. Garoufalis, C.S.; Zdetsis, A.D. High accuracy calculations of the optical gap and absorption spectrum of oxygen contaminated Si nanocrystals. Phys. Chem. Chem. Phys. 2006, 8, 808-813.

70. Dohnalova, K.; Kusova, K.; Pelant, I. Time-resolved photoluminescence spectroscopy of the initial oxidation stage of small silicon nanocrystals. Appl. Phys. Lett. 2009, 94, 211903. 
71. Averboukh, B.; Huber, R.; Cheah, K.W.; Shen, Y.R.; Qin, G.G.; Ma, Z.C.; Zong, W.H. Luminescence studies of a $\mathrm{Si} / \mathrm{SiO}_{2}$ superlattice. J. Appl. Phys. 2002, 92, 3564-3568.

72. Iacona, F.; Bongiorno, C.; Spinella, C.; Boninelli, S.; Priolo, F. Formation and evolution of luminescent $\mathrm{Si}$ nanoclusters produced by thermal annealing of $\mathrm{SiO}_{x}$ films. J. Appl. Phys. 2004, 95, 3723-3732.

73. Wang, Y.Q.; Smirani, R.; Ross, G.G. The formation mechanism of Si nanocrystals in $\mathrm{SiO}_{2}$. J. Cryst. Growth 2006, 294, 486-489.

74. Naciri, A.E.; Mansour, M.; Johann, L.; Grob, J.J.; Eckert, C. Optical study of Si nanocrystals in $\mathrm{Si} / \mathrm{SiO}_{2}$ layers by spectroscopic ellipsometry. Nucl. Instrum. Meth. B 2004, 216, 167-172.

75. Falcony, C.; Calleja, W.; Aceves, M.; Siqueiros, J.M.; Machorro, R.; CotaAraiza, L.; Soto, G.; Farias, M.H. Characterization of excess $\mathrm{Si}$ in nonstoichiometric $\mathrm{SiO}_{2}$ films by optical and surface analysis techniques. J. Electrochem. Soc. 1997, 144, 379-383.

76. Charvet, S.; Madelon, R.; Gourbilleau, F.; Rizk, R. Ellipsometric spectroscopy study of photoluminescent $\mathrm{Si} / \mathrm{SiO}_{2}$ systems obtained by magnetron co-sputtering. J. Lumin. 1998, 80, 257-261.

77. Daldosso, N.; Melchiorri, M.; Pavesi, L.; Pucker, G.; Gourbilleau, F.; Chausserie, S.; Belarouci, A.; Portier, X.; Dufour, C. Optical losses and absorption cross-section of silicon nanocrystals. J. Lumin. 2006, 121, 344-348.

78. Navarro-Urrios, D.; Riboli, F.; Cazzanelli, M.; Chiasera, A.; Daldosso, N.; Pavesi, L.; Oton, C.J.; Heitmann, J.; Yi, L.X.; Scholz, R.; et al. Birefringence characterization of mono-dispersed silicon nanocrystals planar waveguides. Opt. Mater. 2005, 27, 763-768.

79. Khriachtchev, L.; Navarro-Urrios, D.; Pavesi, L.; Oton, C.J.; Capuj, N.E.; Novikov, S. Spectroscopy of silica layers containing Si nanocrystals: Experimental evidence of optical birefringence. J. Appl. Phys. 2007, 101, 044310.

80. Moreno, J.A.; Garrido, B.; Pellegrino, P.; Garcia, C.; Arbiol, J.; Morante, J.R.; Marie, P.; Gourbilleau, F.; Rizk, R. Size dependence of refractive index of Si nanoclusters embedded in $\mathrm{SiO}_{2}$. J. Appl. Phys. 2005, 98, 013523.

81. Chen, T.P.; Liu, Y.; Tse, M.S.; Fung, S.; Dong, G. Profile of optical constants of $\mathrm{SiO}_{2}$ thin films containing Si nanocrystals. J. Appl. Phys. 2004, 95, 8481-8483.

82. Amans, D.; Callard, S.; Gagnaire, A.; Joseph, J.; Ledoux, G.; Huisken, F. Ellipsometric study of silicon nanocrystal optical constants. J. Appl. Phys. 2003, 93, 4173-4179.

83. Mishra, P.; Jain, K.P. Raman, photoluminescence and optical absorption studies on nanocrystalline silicon. Mat. Sci. Eng. B 2002, 95, 202-213.

84. von Behren, J.; van Buuren, T.; Zacharias, M.; Chimowitz, E.H.; Fauchet, P.M. Quantum confinement in nanoscale silicon: The correlation of size with bandgap and luminescence. Solid State Commun. 1998, 105, 317-322.

85. Khriachtchev, L.; Räsänen, M.; Novikov, S. Efficient wavelength-selective optical waveguiding in a silica layer containing Si nanocrystals. Appl. Phys. Lett. 2003, 83, 3018-3020.

86. Valenta, J.; Ostatnicky, T.; Pelant, I.; Elliman, R.G.; Linnros, J.; Honerlage, B. Microcavity-like leaky mode emission from a planar optical waveguide made of luminescent silicon nanocrystals. J. Appl. Phys. 2004, 96, 5222-5225. 
87. Pelant, I.; Ostatnicky, T.; Valenta, J.; Luterova, K.; Skopalova, E.; Mates, T.; Elliman, R.G. Waveguide cores containing silicon nanocrystals as active spectral filters for silicon-based photonics. Appl. Phys. B 2006, 83, 87-91.

88. Tauc, J.; Grigorov, R.; Vancu, A. Optical properties and electronic structure of amorphous germanium. Phys. Status Solidi B 1966, 15, 627-637.

89. BenChorin, M.; Averboukh, B.; Kovalev, D.; Polisski, G.; Koch, F. Influence of quantum confinement on the critical points of the band structure of Si. Phys. Rev. Lett. 1996, 77, 763-766.

90. Podhorodecki, A.; Zatryb, G.; Misiewicz, J.; Wojcik, J.; Mascher, P. Influence of the annealing temperature and silicon concentration on the absorption and emission properties of $\mathrm{Si}$ nanocrystals. J. Appl. Phys. 2007, 102, 043104.

91. Inokuma, T.; Wakayama, Y.; Muramoto, T.; Aoki, R.; Kurata, Y.; Hasegawa, S. Optical properties of Si clusters and Si nanocrystallites in high-temperature annealed $\mathrm{SiO}_{x}$ films. J. Appl. Phys. 1998, 83, 2228-2234.

92. Thompson, M.O.; Galvin, G.J.; Mayer, J.W.; Peercy, P.S.; Poate, J.M.; Jacobson, D.C.; Cullis, A.G.; Chew, N.G. Melting temperature and explosive crystallization of amorphous silicon during pulsed laser irradiation. Phys. Rev. Lett. 1984, 52, 2360-2363.

93. Koyama, H.; Fauchet, P.M. Laser-induced thermal effects on the optical properties of free-standing porous silicon films. J. Appl. Phys. 2000, 87, 1788-1794.

94. Khriachtchev, L.; Räsänen, M.; Novikov, S. Free-standing silica film containing Si nanocrystals: Photoluminescence, Raman scattering, optical waveguiding, and laser-induced thermal effects. Appl. Phys. Lett. 2005, 86, 141911.

95. Khriachtchev, L.; Räsänen, M.; Novikov, S. Continuous-wave laser annealing of free-standing $\mathrm{Si} / \mathrm{SiO}_{2}$ superlattice: Modification of optical, structural, and light-emitting properties. J. Appl. Phys. 2006, 100, 053502.

96. Khriachtchev, L.; Novikov, S. Laser-induced thermal effects on $\mathrm{Si} / \mathrm{SiO}_{2}$ free-standing superlattices. Appl. Phys. A 2007, 87, 761-766.

97. Koyama, H.; Fauchet, P.M. Very large continuous-wave-laser-induced optical absorption in porous silicon films: Evidence for thermal effects. Appl. Phys. Lett. 1998, 73, 3259-3261.

98. Mchedlidze, T.; Arguirov, T.; Kouteva-Arguirova, S.; Kittler, M. Light induced solid-phase crystallization of $\mathrm{Si}$ nanolayers in $\mathrm{Si} / \mathrm{SiO}_{2}$ multiple quantum wells. J. Appl. Phys. 2010, 107, 124302.

99. Khriachtchev, L.; Ossicini, S.; Iacona, F.; Gourbilleau, F. Silicon nanoscale materials: From theoretical simulations to photonic applications. Int. J. Photoenergy 2012, 2012, 872576.

100. Daldosso, N.; Luppi, M.; Ossicini, S.; Degoli, E.; Magri, R.; Dalba, G.; Fornasini, P.; Grisenti, R.; Rocca, F.; Pavesi, L.; et al. Role of the interface region on the optoelectronic properties of silicon nanocrystals embedded in $\mathrm{SiO}_{2}$. Phys. Rev. B 2003, 68, 085327.

101. Djurabekova, F.; Nordlund, K. Atomistic simulation of the interface structure of Si nanocrystals embedded in amorphous silica. Phys. Rev. B 2008, 77, 115325.

102. Luppi, M.; Ossicini, S. Multiple SiO bonds at the silicon cluster surface. J. Appl. Phys. 2003, 94, 2130-2132.

103. Vasiliev, I.; Chelikowsky, J.R.; Martin, R.M. Surface oxidation effects on the optical properties of silicon nanocrystals. Phys. Rev. B 2002, 65, 121302. 
104. Puzder, A.; Williamson, A.J.; Grossman, J.C.; Galli, G. Computational studies of the optical emission of silicon nanocrystals. J. Am. Chem. Soc 2003, 125, 2786-2791.

105. Pennycook, T.J.; Hadjisavvas, G.; Idrobo, J.C.; Kelires, P.C.; Pantelides, S.T. Optical gaps of free and embedded Si nanoclusters: Density functional theory calculations. Phys. Rev. B 2010, 82,125310 .

106. Luppi, E.; Iori, F.; Magri, R.; Pulci, O.; Ossicini, S.; Degoli, E.; Olevano, V. Excitons in silicon nanocrystallites: The nature of luminescence. Phys. Rev. B 2007, 75, 033303.

107. Kleovoulou, K.; Kelires, P.C. Stress state of embedded Si nanocrystals. Phys. Rev. B 2013, $88,085424$.

108. Hadjisavvas, G.; Kelires, P.C. Structure and energetics of Si nanocrystals embedded in a-SiO 2. Phys. Rev. Lett. 2004, 93, 226104.

109. Kleovoulou, K.; Kelires, P.C. Local rigidity and physical trends in embedded Si nanocrystals. Phys. Rev. B 2013, 88, 245202.

110. Guerra, R.; Degoli, E.; Ossicini, S. Size, oxidation, and strain in small $\mathrm{Si} / \mathrm{SiO}_{2}$ nanocrystals. Phys. Rev. B 2009, 80, 155332.

111. Watanabe, T.; Tatsumura, K.; Ohdomari, I. $\mathrm{SiO}_{2} / \mathrm{Si}$ interface structure and its formation studied by large-scale molecular dynamics simulation. Appl. Surf. Sci. 2004, 237, 125-133.

112. Guerra, R.; Marri, I.; Magri, R.; Martin-Samos, L.; Pulci, O.; Degoli, E.; Ossicini, S. Silicon nanocrystallites in a $\mathrm{SiO}_{2}$ matrix: Role of disorder and size. Phys. Rev. B 2009, 79, 155320.

113. Guerra, R.; Marri, I.; Magri, R.; Martin-Samos, L.; Pulci, O.; Degoli, E.; Ossicini, S. Optical properties of silicon nanocrystallites in $\mathrm{SiO}_{2}$ matrix: Crystalline vs. amorphous case. Superlattices Microstruct. 2009, 46, 246-253.

114. Guerra, R.; Ossicini, S. High luminescence in small $\mathrm{SiO} / \mathrm{SiO}_{2}$ nanocrystals: A theoretical study. Phys. Rev. B 2010, 81, 245307.

115. Khriachtchev, L.; Nikitin, T.; Oton, C.J.; Velagapudi, R.; Sainio, J.; Lahtinen, J.; Novikov, S. Optical properties of silicon nanocrystals in silica: Results from spectral filtering effect, $m$-line technique, and X-ray photoelectron spectroscopy. J. Appl. Phys. 2008, 104, 104316.

116. Nikitin, T.; Velagapudi, R.; Sainio, J.; Lahtinen, J.; Räsänen, M.; Novikov, S.; Khriachtchev, L. Optical and structural properties of $\mathrm{SiO}_{x}$ films grown by molecular beam deposition: Effect of the Si concentration and annealing temperature. J. Appl. Phys. 2012, 112, 094316.

117. Khriachtchev, L.; Nikitin, T.; Velagapudi, R.; Lahtinen, J.; Novikov, S. Light-emission mechanism of thermally annealed silicon-rich silicon oxide revisited: What is the role of silicon nanocrystals? Appl. Phys. Lett. 2009, 94, 043115.

118. Nikitin, T.; Aitola, K.; Novikov, S.; Räsänen, M.; Velagapudi, R.; Sainio, J.; Lahtinen, J.; Mizohata, K.; Ahlgren, T.; Khriachtchev, L. Optical and structural properties of silicon-rich silicon oxide films: Comparison of ion implantation and molecular beam deposition methods. Phys. Status Solidi A 2011, 208, 2176-2181.

119. Hartstein, A.; Tsang, J.C.; Dimaria, D.J.; Dong, D.W. Observation of amorphous silicon regions in silicon-rich silicon dioxide films. Appl. Phys. Lett. 1980, 36, 836-837.

120. Khriachtchev, L.; Kilpela, O.; Karirinne, S.; Keranen, J.; Lepisto, T. Substrate-dependent crystallization and enhancement of visible photoluminescence in thermal annealing of $\mathrm{Si} / \mathrm{SiO}_{2}$ superlattices. Appl. Phys. Lett. 2001, 78, 323-325. 
121. Faraci, G.; Gibilisco, S.; Russo, P.; Pennisi, A.R.; La Rosa, S. Modified Raman confinement model for Si nanocrystals. Phys. Rev. B 2006, 73, 033307.

122. Campbell, I.H.; Fauchet, P.M. The effects of microcrystal size and shape on the one phonon Raman spectra of crystalline semiconductors. Solid State Commun. 1986, 58, 739-741.

123. Khriachtchev, L.; Novikov, S.; Kilpela, O. Optics of $\mathrm{Si} / \mathrm{SiO}_{2}$ superlattices: Application to Raman scattering and photoluminescence measurements. J. Appl. Phys. 2000, 87, 7805-7813.

124. Seino, K.; Bechstedt, F.; Kroll, P. Influence of $\mathrm{SiO}_{2}$ matrix on electronic and optical properties of Si nanocrystals. Nanotechnology 2009, 20, 135702.

125. Novikov, S.; Sinkkonen, J.; Nikitin, T.; Khriachtchev, L.; Räsänen, M.; Haimi, E. Free-standing $\mathrm{SiO}_{2}$ films containing $\mathrm{Si}$ nanocrystals directly suitable for transmission electron microscopy. Microelectron. J. 2008, 39, 518-522.

126. Kogelnik, H.; Ramaswamy, V. Scaling rules for thin-film optical waveguides. Appl. Opt. 1974, $13,1857-1862$.

127. Unger, H.G. Planar Optical Waveguides and Fibres; Oxford University Press: Oxford, UK, 1977.

128. Yurtsever, A.; Weyland, M.; Muller, D.A. Three-dimensional imaging of nonspherical silicon nanoparticles embedded in silicon oxide by plasmon tomography. Appl. Phys. Lett. 2006, 89, 151920.

129. Zatryb, G.; Podhorodecki, A.; Misiewicz, J.; Cardin, J.; Gourbilleau, F. Correlation between matrix structural order and compressive stress exerted on silicon nanocrystals embedded in silicon-rich silicon oxide. Nanoscale Res. Lett. 2013, 8, 40.

130. Munekuni, S.; Yamanaka, T.; Shimogaichi, Y.; Tohmon, R.; Ohki, Y.; Nagasawa, K.; Hama, Y. Various types of nonbridging oxygen hole center in highpurity silica glass. J. Appl. Phys. 1990, $68,1212-1217$.

131. Glinka, Y.D.; Lin, S.H.; Hwang, L.P.; Chen, Y.T. Photoluminescence from mesoporous silica: Similarity of properties to porous silicon. Appl. Phys. Lett. 2000, 77, 3968-3970.

132. Borsella, E.; D'Amato, R.; Fabbri, F.; Falconieri, M.; Trave, E.; Bello, V.; Mattei, G.; Nie, Y.R.; Wang, D.Y. On the role of non-bridging oxygen centers in the red luminescence emission from silicon nanocrystals. Phys. Status Solidi C 2011, 8, 974-978.

133. Koponen, L.; Tunturivuori, L.O.; Puska, M.J.; Nieminen, R.M. Effect of the surrounding oxide on the photoabsorption spectra of Si nanocrystals. Phys. Rev. B 2009, 79, 235332.

134. Garcia, C.; Garrido, B.; Pellegrino, P.; Ferre, R.; Moreno, J.A.; Pavesi, L.; Cazzanelli, M.; Morante, J.R. Absorption cross-sections and lifetimes as a function of size in Si nanocrystals embedded in $\mathrm{SiO}_{2}$. Phys. E 2003, 16, 429-433.

135. Shimizu-Iwayama, T.; Kurumado, N.; Hole, D.E.; Townsend, P.D. Optical properties of silicon nanoclusters fabricated by ion implantation. J. Appl. Phys. 1998, 83, 6018-6022.

136. Lee, B.G.; Hiller, D.; Luo, J.W.; Semonin, O.E.; Beard, M.C.; Zacharias, M.; Stradins, P. Strained interface defects in silicon nanocrystals. Adv. Funct. Mater. 2012, 22, 3223-3232.

137. Kusova, K.; Ondic, L.; Klimesova, E.; Herynkova, K.; Pelant, I.; Danis, S.; Valenta, J.; Gallart, M.; Ziegler, M.; Honerlage, B., et al. Luminescence of free-standing versus matrix-embedded oxide-passivated silicon nanocrystals: The role of matrix-induced strain. Appl. Phys. Lett. 2012, 101, 143101. 
138. Faraci, G.; Gibilisco, S.; Pennisi, A.R. Quantum confinement and thermal effects on the Raman spectra of Si nanocrystals. Phys. Rev. B 2009, 80, 193410.

139. Balkanski, M.; Wallis, R.F.; Haro, E. Anharmonic effects in light scattering due to optical phonons in silicon. Phys. Rev. B 1983, 28, 1928-1934.

140. Khriachtchev, L.; Nikitin, T.; Räsänen, M.; Domanskaya, A.; Boninelli, S.; Iacona, F.; Engdahl, A.; Juhanoja, J.; Novikov, S. Continuous-wave laser annealing of Si-rich oxide: A microscopic picture of macroscopic $\mathrm{Si}_{-} \mathrm{SiO}_{2}$ phase separation. J. Appl. Phys. 2010, 108, 124301.

141. Nikitin, T.; Kemell, M.; Puukilainen, E.; Boninelli, S.; Iacona, F.; Räsänen, M.; Ritala, M.; Novikov, S.; Khriachtchev, L. Surface fingerprints of individual silicon nanocrystals in laser-annealed $\mathrm{Si} / \mathrm{SiO}_{2}$ superlattice: Evidence of nanoeruptions of laser-pressurized silicon. J. Appl. Phys. 2012, 111, 124302.

142. Nikitin, T.; Kemell, M.; Puukilainen, E.; Räsänen, M.; Ritala, M.; Novikov, S.; Khriachtchev, L. Continuous-wave laser annealing of a $\mathrm{Si} / \mathrm{SiO}_{2}$ superlattice: Effect of the ambient atmosphere and exposure period. Sci. Adv. Mater. 2014, 6, 1000-1010.

143. Bachels, T.; Schafer, R. Binding energies of neutral silicon clusters. Chem. Phys. Lett. 2000, 324, $365-372$.

144. Murphy, D.V.; Brueck, S.R.J. Enhanced raman-scattering from silicon microstructures. Opt. Lett. 1983, 8, 494-496.

145. Doremus, R.H. Viscosity of silica. J. Appl. Phys. 2002, 92, 7619-7629.

146. Wada, K.; Suzuki, A.; Sato, H.; Kikuchi, R. Soret effect in solids. J. Phys. Chem. Solids 1985, 46, 1195-1205.

147. Zhang, K.J.; Briggs, M.E.; Gammon, R.W.; Sengers, J.V. Optical measurement of the Soret coefficient and the diffusion coefficient of liquid mixtures. J. Chem. Phys. 1996, 104, 6881-6892.

148. Susa, M.; Nagata, K. Thermal oxidation of silicon substrates through oxygen diffusion. Mat. Sci. Eng. A 1991, 146, 51-62.

149. Deal, B.E.; Grove, A.S. General relationship for thermal oxidation of silicon. J. App. Phys. 1965, 36, 3770-3778.

150. Lukes, F.; Schmidt, E. Oxidation of silicon in dry oxygen. J. Phys. Chem. Solids 1965, 26, 1353-1357.

151. Nikitin, T.; Khriachtchev, L.; Räsänen, M.; Novikov, S. Optical memory of silicon nanocrystals with submicron spatial resolution and very high thermal stability. Appl. Phys. Lett. 2009, 94, 173116.

152. Khriachtchev, L. Comment on Optical absorption measurements of silica containing Si nanocrystals produced by ion implantation and thermal annealing. Appl. Phys. Lett. 2002, 81, 1357-1358.

153. Oton, C.J.; Ghulinyan, M.; Gaburro, Z.; Bettotti, P.; Pavesi, L.; Pancheri, L.; Gialanella, S.; Capuj, N.E. Scattering rings as a tool for birefringence measurements in porous silicon. J. Appl. Phys. 2003, 94, 6334-6340.

154. Renault, O.; Marlier, R.; Gely, M.; De Salvo, B.; Baron, T.; Hansson, M.; Barrett, N.T. Synchrotron radiation X-ray photoelectron spectroscopy of Si nanocrystals grown onto $\mathrm{Al}_{2} \mathrm{O}_{3} / \mathrm{Si}$ surfaces. Appl. Phys. Lett. 2005, 87, 163119. 
155. Kim, S.; Kim, M.C.; Choi, S.H.; Kim, K.J.; Hwang, H.N.; Hwang, C.C. Size dependence of Si $2 p$ core-level shift at $\mathrm{Si}$ nanocrystal/ $/ \mathrm{SiO}_{2}$ interfaces. Appl. Phys. Lett. 2007, 91, 103113.

156. Barbagiovanni, E.G.; Goncharova, L.V.; Simpson, P.J. Electronic structure study of ion-implanted Si quantum dots in $\mathrm{SiO}_{2}$ matrix: Analysis of quantum confinement theories. Phys. Rev. B 2011, 83, 035112.

157. Bruggeman, D.A.G. Berechnung verschiedener physikalischer Konstanten von heterogenen Substanzen. I. Dielektrizitätskonstanten und Leitfähigkeiten der Mischkörper aus isotropen Substanzen. Ann. Phys. 1935, 416, 636-664.

158. Knief, S.; von Niessen, W. Disorder, defects, and optical absorption in a-Si and a-Si:H. Phys. Rev. B 1999, 59, 12940-12946.

(C) 2015 by the authors; licensee MDPI, Basel, Switzerland. This article is an open access article distributed under the terms and conditions of the Creative Commons Attribution license (http://creativecommons.org/licenses/by/4.0/). 